\title{
On invariant structures of black hole charges
}

\author{
Sergio Ferrara, ${ }^{a, b, c}$ Alessio Marrani $^{a}$ and Armen Yeranyan ${ }^{b, d}$ \\ ${ }^{a}$ Physics Department, Theory Unit, CERN, \\ CH -1211, Geneva 23, Switzerland \\ ${ }^{b}$ INFN - Laboratori Nazionali di Frascati, \\ Via Enrico Fermi 40, I-00044 Frascati, Italy \\ ${ }^{c}$ Department of Physics and Astronomy, University of California, \\ Los Angeles, CA 90095-1547, U.S.A. \\ ${ }^{d}$ Department of Physics, Yerevan State University, \\ Alex Manoogian St. 1, Yerevan, 0025, Armenia \\ E-mail: sergio.ferrara@cern.ch, alessio.marrani@cern.ch, \\ ayeran@lnf .infn.it
}

ABStRact: We study "minimal degree" complete bases of duality- and "horizontal"- invariant homogeneous polynomials in the flux representation of two-centered black hole solutions in two classes of $D=4$ Einstein supergravity models with symmetric vector multiplets' scalar manifolds. Both classes exhibit an SL $(2, \mathbb{R})$ "horizontal" symmetry which mixes the two centers.

The first class encompasses $\mathcal{N}=2$ and $\mathcal{N}=4$ matter-coupled theories, with semisimple $U$-duality given by $\mathrm{SL}(2, \mathbb{R}) \times \mathrm{SO}(m, n)$; the analysis is carried out in the socalled Calabi-Vesentini symplectic frame (exhibiting maximal manifest covariance) and until order six in the fluxes included.

The second class, exhibiting a non-trivial "horizontal" stabilizer $\mathrm{SO}(2)$, includes $\mathcal{N}=2$ minimally coupled and $\mathcal{N}=3$ matter coupled theories, with $U$-duality given by the pseudounitary group $\mathrm{U}(r, s)$ (related to complex flux representations).

Finally, we comment on the formulation of special Kähler geometry in terms of "generalized" groups of type $E_{7}$.

KEYwORDS: Black Holes in String Theory, Supergravity Models, Extended Supersymmetry

ARXIV EPRINT: 1110.4004 


\section{Contents}

1 Introduction 1

2 Calabi-Vesentini flux tensors and invariants 4

2.1 Order 2

2.2 Order $4 \quad 6$

$\begin{array}{lll}2.2 .1 & \text { Summary } & 12\end{array}$

2.3 Order $6 \quad 12$

2.3.1 Summary 14

3 Invariants of pseudo-unitary $U$-duality $\quad \mathbf{1 5}$

3.1 $\mathrm{SL}(p, \mathbb{C}) \times \mathrm{U}(r, s) \quad 16$

$\begin{array}{ll}3.2 \mathrm{SL}_{h}(2, \mathbb{R}) \times \mathrm{U}(r, s) & 17\end{array}$

4 Remarks 19

$\begin{array}{lll}4.1 & \text { On CV models } & 19\end{array}$

4.2 On pseudo-unitary $U$-duality 20

4.3 On special geometry and "Generalized" groups of type $E_{7} \quad 21$

\section{Introduction}

We consider reducible symmetric supergravity models in $D=4$ space-time dimensions, ${ }^{1}$ which we will here dub Calabi-Vesentini $(C V)$ models, for reasons which will be evident from treatment below. As also given by table 1, these models are characterized by the following $U$-duality ${ }^{2}$ and "horizontal" [9] symmetries:

$$
\begin{aligned}
& U \text {-duality : } G_{4}=\mathrm{SL}_{v}(2, \mathbb{R}) \times \mathrm{SO}(m, n-2) ; \\
& \text { "horizontal" }: \mathcal{G}_{p}=\mathrm{SL}_{h}(p, \mathbb{R}),
\end{aligned} \quad m=\left\{\begin{array}{l}
2(\mathcal{N}=2, n \geqslant 3) ; \\
6(\mathcal{N}=4, n \geqslant 2),
\end{array}\right.
$$

where $p$ denotes the number of centers of the multi-centered solution under consideration.

Considering an array of $p$ charge vectors $\mathcal{Q}_{a}^{M}(a=1, \ldots, p)$ pertaining to a $p$-centered solution, the $U$-duality group acts on the index $M$ in a symplectic representation:

$$
\mathcal{Q}_{a}^{M} \rightarrow \mathcal{S}_{P}^{M} \mathcal{Q}_{a}^{P}, \quad \mathcal{S}^{T} \mathbb{C} \mathcal{S}=\mathbb{C}
$$

\footnotetext{
${ }^{1}$ Marginal stability for these models was studied e.g. in $[1,5]$.

${ }^{2}$ Here $U$-duality is referred to as the "continuous" symmetries of [6]. Their discrete versions are the $U$-duality non-perturbative string theory symmetries introduced by Hull and Townsend [8].
} 
where $\mathbb{C}_{M N}$ is the symplectic-invariant metric (defined in (2.87) below). On the other hand, the "horizontal" symmetry acts on the index $a$ as a linear transformation on the $p$ vectors:

$$
\mathcal{Q}_{a}^{M} \rightarrow L_{a}^{b} \mathcal{Q}_{b}^{M}, \quad L \in \mathrm{SL}_{h}(p, \mathbb{R}) .
$$

The "horizontal" symmetry, which is not a symmetry of the Lagrangian formulation of the theory, proves to be useful in the classification of multi-charge orbits, which are relevant for the dynamics of multi-centered (black hole) solutions in supergravity [9-11]. For the two-centered case $(p=2)$ considered in the present investigation, the lowest-order dualityand "horizontal"- invariant polynomial is of order 2 in the charges, and it is nothing but the usual Schwinger symplectic product $\mathcal{W}$ of two dyonic charge vectors (see (2.16) below).

As evident from (1.1), we anticipate that the case of $\mathcal{N}=4$ theory coupled to $n_{V, \mathcal{N}=4}=n-2 \geqslant 0$ matter (vector) multiplets can be recovered by shifting $n \rightarrow n+4$ in all formulæ of the treatment below. The semi-simple nature of $G_{4}$ justifies the name "reducible", whereas "symmetric" is due to the fact that the corresponding scalar manifolds belong to the sequence $\mathcal{S} \mathcal{T}[m, n]$, of particular relevance for superstring compactifications (see e.g. the analysis in section 3.1 and appendix $\mathrm{C}$ of [12], and refs. therein).

Let us now reconsider the " $T$-tensor formalism" for $\mathrm{CV}$ models, introduced in sections 3 and 4 of [9], which will be further extended, until order 6 included, in section 2. A key feature of $\mathrm{CV}$ models is the fact that the electro-magnetic splitting

$$
\mathcal{Q}^{M} \equiv\left(p^{\Lambda}, q_{\Lambda}\right)
$$

of the symplectic vector of the 2-form field strengths' fluxes (also named magnetic and electric charges) can be implemented with full manifest covariance with respect to $G_{4}(1.1)$. Namely, $\mathcal{Q}$ sits in the $(\mathbf{2}, \mathbf{m}+\mathbf{n}-\mathbf{2})$ bi-fundamental irrep. of $G_{4}$, and it is thus an electromagnetic doublet $\mathbf{2}$ of the "vertical" $\mathrm{SL}_{v}(2, \mathbb{R})$; the symplectic index $M$ thus splits as follows (cfr. eq. (3.7) of [9])

$$
\left.\begin{array}{l}
\begin{array}{l}
M=\alpha \Lambda, \\
\alpha=1,2, \Lambda=1, \ldots, m+n-2 .
\end{array}
\end{array}\right\} \Rightarrow \mathcal{Q}^{M} \equiv \mathcal{Q}_{\alpha}^{\Lambda},
$$

and it should be pointed out that in the $\mathcal{N}=2$ case usually $\Lambda=0,1, \ldots, n-1$, with "0" pertaining to the $D=4$ graviphoton vector. The manifestly $G_{4}$-covariant symplectic frame (1.5) is usually dubbed Calabi-Vesentini frame [15], and it was firstly introduced in supergravity in [16].

By defining

$$
p^{2} \equiv p^{\Lambda} p^{\Sigma} \eta_{\Lambda \Sigma}, q^{2} \equiv q_{\Lambda} q_{\Sigma} \eta^{\Lambda \Sigma}, p \cdot q \equiv p^{\Lambda} q_{\Lambda},
$$

where $\eta_{\Lambda \Sigma}=\eta^{\Lambda \Sigma}$ is the pseudo-Euclidean metric of SO $(m, n-2)$, the unique algebraicallyindependent single-centered $G_{4}$-invariant polynomial $I_{4}$ (homogeneous of order 4 in the fluxes) reads [17-20]

$$
I_{4}(\mathcal{Q}) \equiv p^{2} q^{2}-(p \cdot q)^{2},
$$

and, by virtue of the CV covariant split (1.5), it can be rewritten as:

$$
I_{4}(\mathcal{Q})=\frac{1}{2} \epsilon^{\alpha \beta} \epsilon^{\gamma \delta} \eta_{\Lambda \Xi \eta_{\Sigma \Omega}} \mathcal{Q}_{\alpha}^{\Lambda} \mathcal{Q}_{\beta}^{\Sigma} \mathcal{Q}_{\gamma}^{\Xi} \mathcal{Q}_{\delta}^{\Omega} \equiv \frac{1}{2} \mathbb{K}_{\Lambda \Sigma \Xi \Omega}^{\alpha \beta \gamma \delta} \mathcal{Q}_{\alpha}^{\Lambda} \mathcal{Q}_{\beta}^{\Sigma} \mathcal{Q}_{\gamma}^{\Xi} \mathcal{Q}_{\delta}^{\Omega},
$$




\begin{tabular}{|l|l|c|c|}
\hline $\mathcal{N}$ & $\frac{G_{4}}{m c s\left(G_{4}\right)}$ & rank & $\begin{array}{c}J_{3} \\
\text { reducible }\end{array}$ \\
\hline 2 & $\frac{\mathrm{SL}_{v}(2, \mathbb{R})}{\mathrm{U}(1)} \times \frac{\mathrm{SO}(2, n-2)}{\mathrm{SO}(2) \times \mathrm{SO}(n-2)}, n \geqslant 3$ & $1+\min (2, n-2)$ & $\mathbb{R} \oplus \boldsymbol{\Gamma}_{1, n-3}$ \\
\hline 4 & $\frac{\mathrm{SL}_{v}(2, \mathbb{R})}{\mathrm{U}(1)} \times \frac{\mathrm{SO}(6, n-2)}{\mathrm{SO}(6) \times \mathrm{SO}(n-2)}, n \geqslant 2$ & $1+\min (6, n-2)$ & $\mathbb{R} \oplus \boldsymbol{\Gamma}_{5, n-3}$ \\
\hline
\end{tabular}

Table 1. Calabi-Vesentini $d=4$ supergravity models. "mcs" stands for maximal compact subgroup (with symmetric embedding). The rank of the scalar manifold, as well as the related reducible Euclidean rank-3 Jordan algebra $J_{3}$ are also given (for further elucidation and a recent treatment, see e.g. $[13,14]$ and refs. therein). The subscript " $v$ " stands for "vertical", and it has been introduced in order to distinguish the $S$-duality $\mathrm{SL}_{v}(2, \mathbb{R})$ group from the "horizontal" symmetry group $\mathrm{SL}_{h}(2, \mathbb{R})$.

where $\mathbb{K}_{\Lambda \Sigma \Xi \Omega \Omega}^{\alpha \beta \gamma \delta}$ is the $G_{4}$-invariant rank-4 completely symmetric $\mathbb{K}$-tensor $\mathbb{K}_{M N P Q}$ (see e.g. [21] and refs. therein) of the CV models, which enjoys the reducible expression

$$
\mathbb{K}_{\Lambda \Sigma \Xi \Omega}^{\alpha \beta \gamma \delta} \equiv \frac{1}{6}\left[\left(\epsilon^{\alpha \beta} \epsilon^{\gamma \delta}+\epsilon^{\alpha \delta} \epsilon^{\beta \gamma}\right) \eta_{\Lambda \Xi} \eta_{\Sigma \Omega}+\left(\epsilon^{\alpha \beta} \epsilon^{\delta \gamma}+\epsilon^{\alpha \gamma} \epsilon^{\delta \beta}\right) \eta_{\Lambda \Omega} \eta_{\Sigma \Xi}+\left(\epsilon^{\alpha \gamma} \epsilon^{\beta \delta}+\epsilon^{\alpha \delta} \epsilon^{\beta \gamma}\right) \eta_{\Lambda \Sigma} \eta_{\Xi \Omega}\right]
$$

in term of the invariant structures $\epsilon^{\alpha \beta}$ and $\eta_{\Lambda \Xi}$ of $\mathrm{SL}_{v}(2, \mathbb{R})$ and of $\mathrm{SO}(m, n-2)$, respectively.

We recall that the rank-2 antisymmetric $T$-tensor

$$
T^{\Lambda \Sigma} \equiv p^{\Lambda} q^{\Sigma}-q^{\Lambda} p^{\Sigma}
$$

plays a key role in the classification of single-centered black hole (BH) charge orbits in CV models (see e.g. [22-25]); furthermore, we anticipate that $T^{\Lambda \Sigma}(1.10)$ is the "1-centered limit" $1 \equiv 2$ of the tensor $T_{(a b)}^{[\Lambda \Sigma]}$ defined by the third of (2.6) further below, relevant for the treatment of 2-centered BH solutions. As it is well known, the order-4 invariant $\mathcal{I}_{4}(\mathcal{Q})(1.7)-(1.8)$ also enjoys a simple expression in terms of the tensor $T^{\Lambda \Sigma}(1.10)$ :

$$
I_{4}(\mathcal{Q})=\frac{1}{2} T^{\Lambda \Sigma} T^{\Xi \Omega} \eta_{\Lambda \Xi \eta_{\Sigma \Omega}}=-\frac{1}{2} \operatorname{Tr}_{\eta}\left(\mathbb{T}^{2}\right)
$$

where " $\operatorname{Tr}_{\eta}$ " denotes throughout the $\eta$-trace, namely the trace in which the $\mathrm{SO}(m, n-2)$ vector indices are consistently raised and lowered by the $\eta$-structure.

From (1.1), the ("horizontal" $\times U$-duality) group of a 2-centered solution in $D=4$ CV models reads

$$
\mathcal{G}_{p=2} \times G_{4}=\mathrm{SL}_{h}(2, \mathbb{R}) \times \mathrm{SL}_{v}(2, \mathbb{R}) \times \mathrm{SO}(m, n-2) \sim \mathrm{SO}(2,2)_{h}^{v} \times \mathrm{SO}(m, n-2),
$$

where we recall that " $h$ " and " $v$ " respectively stand for "horizontal" and "vertical". In the $\mathcal{N}=2$ case, the number of (Abelian) vector multiplets coupled to the gravity multiplet is 
$n_{V, \mathcal{N}=2}=n-1$. We will throughout consider 2-centered 0-brane (BH) solutions, and thus the relevant representation of $\mathcal{G}_{p=2} \times G_{4}$ in which the corresponding 2-form field strengths fluxes $\mathcal{Q}_{a \alpha}^{\Lambda}$ sit is

$$
(\mathbf{2}, \mathbf{2}, \mathbf{n}) \text { of } \mathrm{SL}_{h}(2, \mathbb{R}) \times \mathrm{SL}_{v}(2, \mathbb{R}) \times \mathrm{SO}(2, n-2),
$$

which is thus amenable to a Gramian treatment, as considered in section 8 of [9]. In the following treatment, the $\mathbf{2}$ of $\mathrm{SL}_{h}(2, \mathbb{R})$ is spanned by the Latin lowercase indices $a=1,2$, the $\mathbf{2}$ of $\mathrm{SL}_{v}(2, \mathbb{R})$ is spanned by the Greek lowercase indices $\alpha=1,2$, and the vector $\mathbf{n}$ of $\mathrm{SO}(2, n-2)$ is spanned by the uppercase Greek indices $\Lambda=0,1, \ldots, n-1$ (" 0 " pertaining to the graviphotonic fluxes, as mentioned above). If no further decomposition with respect to proper subgroups is considered, maximal $G_{4}$-covariance is manifest, and, as stated, the symplectic frame under consideration is usually dubbed CV [15] frame [16].

Aim of the present note is to give a complete treatment of 2 -centered $G_{4^{-}}$("duality") and $\left(\mathcal{G}_{p=2} \times G_{4}\right)$ - (dubbed "horizontal") invariant homogeneous polynomial structures in the CV symplectic frame at order 2, 4 and 6 in the fluxes, thus clarifying, generalizing and completing the treatment given in [9], whose notation and formulæ we will often refer to (reporting some of them, for ease of consultation). We will also briefly comment on the 2-centered "horizontal" symmetry of supergravity models with pseudo-unitary $U$-duality groups, refining the analysis of [26].

The plan of the note is as follows.

In section 2 we analyze the duality- and "horizontal"- invariant two-centered homogeneous polynomials in Calabi-Vesentini $D=4$ supergravity models, at order 2 (section 2.1), 4 (2.2) and 6 (2.3) in the fluxes $\mathcal{Q}$ 's, which is enough to determine the corresponding complete "minimal degree" bases (see discussion in section 4.1).

Then, in section 3 we study duality- and "horizontal"- invariant two-centered polynomials in $D=4$ symmetric supergravity models with $U$-duality group $G_{4}$ given by the pseudo-unitary group $\mathrm{U}(r, s)$.

The final section 4 contains various remarks and observations, concerning the CV models (section 4.1) and models with $G_{4}=\mathrm{U}(r, s)$ (section 4.2). Moreover, in section 4.3, by suitably generalizing the notion of groups "of type $E_{7}$ " [24, 28, 29], we comment on their relation to special Kähler geometry.

\section{Calabi-Vesentini flux tensors and invariants}

\section{$2.1 \quad$ Order 2}

We start and consider the rank-2 tensor product

$$
\mathcal{Q}_{a \alpha}^{\Lambda} \mathcal{Q}_{b \beta}^{\Sigma} \equiv(\underset{a}{\mathbf{2}}, \underset{\alpha}{\mathbf{2}}, \underset{\Lambda}{\mathbf{n}}) \times_{s}(\underset{b}{\mathbf{2}}, \underset{\beta}{\mathbf{2}}, \underset{\Sigma}{\mathbf{n}})=\left(\mathbf{3}_{s}+\mathbf{1}_{a}, \mathbf{3}_{s}+\mathbf{1}_{a},\left[\frac{\mathbf{n}(\mathbf{n}+\mathbf{1})}{\mathbf{2}}\right]_{s}+\left[\frac{\mathbf{n}(\mathbf{n}-\mathbf{1})}{\mathbf{2}}\right]_{a}\right)_{s},
$$

where "s" and " $a$ " denote the symmetric and antisymmetric parts throughout. Ça va sans dire, the obvious symmetry of $\mathcal{Q}_{a \alpha}^{\Lambda} \mathcal{Q}_{b \beta}^{\Sigma}$ under the exchange $a \alpha \Lambda \leftrightarrow b \beta \Sigma$ restricts the analysis to the symmetric part of such a tensor product. Since in (pseudo-)orthogonal groups 
the symmetric rank-2 repr. can be further irreducibly split in traceless $\mathbf{S}_{0}$ and trace $\mathbf{1}$ irreps. (with the naught denoting $\eta$-tracelessness in $\mathrm{SO}(2, n-2)$ throughout; see Footnote 4 of [9]):

$$
\frac{\mathbf{n}(\mathbf{n}+\mathbf{1})}{\mathbf{2}}=\mathbf{S}_{0}+\mathbf{1}
$$

(2.1) can be further elaborated as:

$\mathcal{Q}_{a \alpha}^{\Lambda} \mathcal{Q}_{b \beta}^{\Sigma}=\left(\mathbf{3}, \mathbf{3}, \mathbf{S}_{0}\right)+(\mathbf{3}, \mathbf{3}, \mathbf{1})+(\mathbf{3}, \mathbf{1}, \mathbf{A d j})+(\mathbf{1}, \mathbf{3}, \mathbf{A d j})+\left(\mathbf{1}, \mathbf{1}, \mathbf{S}_{0}\right)+(\mathbf{1}, \mathbf{1}, \mathbf{1})$,

where the following notation for $\mathrm{SO}(2, n-2)$ irreps. has been introduced:

$$
\begin{aligned}
\mathbf{S}_{0} & \equiv \frac{\mathbf{n}(\mathbf{n}+\mathbf{1})}{\mathbf{2}}-\mathbf{1}(\eta \text {-traceless rank-2 symmetric }) \\
\mathbf{A d j} & \equiv \frac{\mathbf{n}(\mathbf{n}-\mathbf{1})}{\mathbf{2}}(\text { rank-2 antisymmetric, i.e. adjoint }) .
\end{aligned}
$$

The total real dimension of $\mathcal{Q}_{a \alpha}^{\Lambda} \mathcal{Q}_{b \beta}^{\Sigma}$ is $2 n(4 n+1)$. Thence, one can assign to each irreps. a tensor structure (with "\#" denoting the corresponding dimension):

$$
\begin{array}{lll}
\left(\mathbf{3}, \mathbf{3}, \mathbf{S}_{0}\right): & T_{(a b)(\alpha \beta)}^{(\Lambda \Sigma)} \equiv T_{(a b)(\alpha \beta)}^{(\Lambda \Sigma)}-\frac{1}{n} \eta^{\Lambda \Sigma} \operatorname{Tr}_{\eta}\left(T_{(a b)(\alpha \beta)}\right), & \#=\frac{9}{2} n(n+1)-1 ; \\
(\mathbf{3}, \mathbf{3}, \mathbf{1}): & \mathbf{T}_{(a b)(\alpha \beta)}^{(\Lambda \Sigma)} \equiv \frac{1}{n} \eta^{\Lambda \Sigma} \operatorname{Tr}_{\eta}\left(T_{(a b)(\alpha \beta)}\right), & \#=1 ; \\
(\mathbf{3}, \mathbf{1}, \mathbf{A d j}): & T_{(a b)[\alpha \beta]}^{[\Lambda \Sigma]} \Rightarrow T_{(a b)}^{[\Lambda \Sigma]} \equiv \epsilon^{\alpha \beta} T_{(a b)[\alpha \beta]}^{[\Lambda \Sigma]}, & \#=\frac{3}{2} n(n+1) ; \\
(\mathbf{1}, \mathbf{3}, \mathbf{A d j}): & T_{[a b](\alpha \beta)}^{[\Lambda \Sigma]} \Rightarrow T_{(\alpha \beta)}^{[\Lambda \Sigma]} \equiv \epsilon^{a b} T_{(a b)[\alpha \beta]}^{[\Lambda \Sigma]}, & \#=\frac{3}{2} n(n+1) ; \\
\left(\mathbf{1}, \mathbf{1}, \mathbf{S}_{0}+\mathbf{1}\right): & T_{[a b][\alpha \beta]}^{(\Lambda \Sigma)} \Rightarrow\left\{\begin{array}{l}
T_{[\alpha \beta]}^{(\Lambda \Sigma)} \equiv \epsilon^{a b} T_{[a b][\alpha \beta]}^{(\Lambda \Sigma)} ; \\
T_{[a b]}^{(\Lambda \Sigma)} \equiv \epsilon^{\alpha \beta} T_{[a b][\alpha \beta]}^{(\Lambda \Sigma)} ; \\
T^{(\Lambda \Sigma)} \equiv \epsilon^{a b} \epsilon^{\alpha \beta} T_{[a b][\alpha \beta]}^{(\Lambda \Sigma)} .
\end{array}\right. & \text { \# } \quad \text { (each of them) }
\end{array}
$$

Below, we will also use the further irreducibly split $\left(\mathbf{1}, \mathbf{1}, \mathbf{S}_{0}+\mathbf{1}\right)$ (as $(2.2)$ ), reading:

$$
\begin{array}{ccc}
\left(\mathbf{1}, \mathbf{1}, \mathbf{S}_{0}\right): & T_{[a b][\alpha \beta]}^{0(\Lambda \Sigma)} \equiv T_{[a b][\alpha \beta]}^{(\Lambda \Sigma)}-\frac{1}{n} \eta^{\Lambda \Sigma} \operatorname{Tr}_{\eta}\left(T_{[a b][\alpha \beta]}\right), \quad \#=\frac{1}{2} n(n+1)-1 ; \\
(\mathbf{1}, \mathbf{1}, \mathbf{1}): & \mathbf{T}_{[a b][\alpha \beta]}^{(\Lambda \Sigma)} \equiv \frac{1}{n} \eta^{\Lambda \Sigma} \operatorname{Tr}_{\eta}\left(T_{[a b][\alpha \beta]}\right), \quad & \#=1 .
\end{array}
$$


Explicit expressions in terms of the flux vector $\mathcal{Q}_{a \alpha}^{\Lambda}$ read: ${ }^{3}$

$$
\begin{aligned}
T_{(a b)(\alpha \beta)}^{(\Lambda \Sigma)} & =\frac{1}{4}\left(\mathcal{Q}_{a \alpha}^{\Lambda} \mathcal{Q}_{b \beta}^{\Sigma}+\mathcal{Q}_{a \alpha}^{\Sigma} \mathcal{Q}_{b \beta}^{\Lambda}+\mathcal{Q}_{a \beta}^{\Lambda} \mathcal{Q}_{b \alpha}^{\Sigma}+\mathcal{Q}_{a \beta}^{\Sigma} \mathcal{Q}_{b \alpha}^{\Lambda}\right) ; \\
T_{(a b)}^{[\Lambda \Sigma]} & =\frac{1}{2}\left(\mathcal{Q}_{a \alpha}^{\Lambda} \mathcal{Q}_{b \beta}^{\Sigma}-\mathcal{Q}_{a \alpha}^{\Sigma} \mathcal{Q}_{b \beta}^{\Lambda}\right) \epsilon^{\alpha \beta} ; \\
T_{(\alpha \beta)}^{[\Lambda \Sigma]} & =\frac{1}{2}\left(\mathcal{Q}_{a \alpha}^{\Lambda} \mathcal{Q}_{b \beta}^{\Sigma}-\mathcal{Q}_{a \beta}^{\Sigma} \mathcal{Q}_{b \alpha}^{\Lambda}\right) \epsilon^{a b} ; \\
T^{(\Lambda \Sigma)} & =\mathcal{Q}_{a \alpha}^{\Lambda} \mathcal{Q}_{b \beta}^{\Sigma} \epsilon^{a b} \epsilon^{\alpha \beta} .
\end{aligned}
$$

We will also make use of the following $\mathrm{SO}(2, n-2)$-matrix notations:

$$
\begin{aligned}
T_{(a b)(\alpha \beta)} & \equiv T_{(a b)(\alpha \beta)}^{(\Lambda \Sigma)} ; \\
T_{(a b)}^{\prime} & \equiv T_{(a b)}^{[\Lambda \Sigma]} ; \\
T_{[a b][\alpha \beta]}^{\prime \prime} & \equiv T_{[a b][\alpha \beta]}^{(\Lambda \Sigma)},
\end{aligned}
$$

and analogous ones for the $\epsilon$-traces.

By further taking (half of) the $\eta$-trace of $T^{(\Lambda \Sigma)}$, one obtains the symplectic product $\mathcal{W}$ of the two charge vectors $\mathcal{Q}_{1 \alpha}^{\Lambda}$ and $\mathcal{Q}_{2 \alpha}^{\Lambda}$ (cfr. e.g. (4.12) of [9], as well as (3.9) of [10]):

$$
\mathcal{W}=\frac{1}{2} \eta_{\Lambda \Sigma} T^{(\Lambda \Sigma)}=\frac{1}{2} \epsilon^{a b} \epsilon^{\alpha \beta} \eta_{\Lambda \Sigma} T_{[a b][\alpha \beta]}^{(\Lambda \Sigma)}=\frac{1}{2} \operatorname{Tr}_{\eta}\left(T^{\prime \prime}\right),
$$

which is evidently "horizontal" (i.e. $\left(\mathcal{G}_{p=2} \times G_{4}\right)$-) invariant (actually, as yielded by the analysis of [9] and [10], the unique "horizontal"-invariant polynomial at order 2 in the fluxes). Clearly, (2.15) is a specification for CV models of the general formula (cfr. e.g. eq. (3.9) of [10], and section 3 therein for notation)

$$
\mathcal{W} \equiv \frac{1}{2} \mathbb{C}_{M N} \epsilon^{a b} \mathcal{Q}_{a}^{M} \mathcal{Q}_{b}^{N},
$$

where $\mathbb{C}_{M N}$ is the symplectic-invariant metric defined in (2.87) below.

The $\epsilon$-traced tensors in (2.6) have been introduced in order to develop the subsequent treatment. Indeed, due to the very structure of (2.2), the irreducible splitting (2.2) is not relevant in order to classify and relate duality- and "horizontal"- invariant homogeneous polynomials in the $\mathrm{BH}$ fluxes (see the treatment of sections 2.2 and 2.3 ).

\section{$2.2 \quad$ Order 4}

Since there is no duality- nor "horizontal"- invariant polynomial structure at order 3 , next we proceed to analyze the order 4 in the fluxes of the 2-centered $\mathrm{BH}$ solution in the framework under consideration. By exploiting the associativity of the (ir)reps.' tensor product, in each of the $\mathrm{SL}_{h}(2, \mathbb{R})$ - and $\mathrm{SL}_{v}(2, \mathbb{R})$ - sectors one gets

$$
\mathbf{2} \times \mathbf{2} \times \mathbf{2} \times \mathbf{2}=\mathbf{3} \times \mathbf{3}+2 \cdot(\mathbf{3} \times \mathbf{1})+\mathbf{1} \times \mathbf{1}=\mathbf{5}+3 \cdot \mathbf{3}+2 \cdot \mathbf{1},
$$

\footnotetext{
${ }^{3}$ In order to make contact with the notation of [9], we observe that $T_{(a b)}^{[\Lambda \Sigma]}(2.9$; also see $(2.13))$ and $T^{(\Lambda \Sigma)}(2.11$; also see $(2.14))$ respectively correspond to $\mathbb{T} \equiv\left(\mathbb{T}_{11} \equiv \mathbb{T}_{1}, \mathbb{T}_{12}, \mathbb{T}_{22} \equiv \mathbb{T}_{2}\right)$ and $2 \mathbb{T}_{a}^{\Lambda \Sigma}$, in turn given by eqs. (3.3)-(3.6) resp. (4.2) of [9].
} 
whereas in the $\mathrm{SO}(2, n-2)$ sector, recalling that

$$
\begin{aligned}
\mathbf{S}_{0} \times \mathbf{S}_{0} & =\mathbf{1}_{s}+\mathbf{A d j}_{a}+\mathbf{S}_{0, s}+\ldots ; \\
\mathbf{A d j} \times \mathbf{A d j} & =\mathbf{1}_{s}+\mathbf{A d j}_{a}+\mathbf{S}_{0, s}+\ldots ; \\
\mathbf{A d j} \times \mathbf{S}_{0} & =\mathbf{A d j}+\mathbf{S}_{0}+\ldots,
\end{aligned}
$$

it holds

$$
\mathbf{n} \times \mathbf{n} \times \mathbf{n} \times \mathbf{n}=\left(\mathbf{S}_{0}+\mathbf{1}+\mathbf{A d j}\right) \times\left(\mathbf{S}_{0}+\mathbf{1}+\mathbf{A d j}\right)=3 \cdot \mathbf{1}+6 \cdot \mathbf{A d j}+6 \cdot \mathbf{S}_{0}+\ldots
$$

Thus, the duality- or "horizontal"- invariant homogeneous polynomials at order 4 in the fluxes arise from the following tensor products:

1.

$$
\left(\mathbf{3}, \mathbf{3}, \mathbf{S}_{0}\right) \times\left(\mathbf{3}, \mathbf{3}, \mathbf{S}_{0}\right)=\left(\underset{G_{4} \text {-inv. }}{\mathbf{3} \times \mathbf{1}, \mathbf{1})+\ldots}\right.
$$

Since $\mathbf{1} \notin \mathbf{S}_{2} \times \mathbf{A d j}$, there are no other sources of duality-invariant polynomials involving tensor products of $\left(\mathbf{3}, \mathbf{3}, \mathbf{S}_{0}\right)$. By using the $\mathrm{SO}(2, n-2)$-matrix notation (2.12), the order-4 $G_{4}$-invariant polynomial from $(2.22)$ can reducibly be rewritten as the $\mathrm{SL}_{h}(2, \mathbb{R})$-bi-triplet

$$
\begin{aligned}
& \mathcal{J}_{(a b)(c d)}^{0} \equiv-T_{(a b)(\alpha \beta)}^{0(\Lambda \Sigma)} T_{(c d)(\gamma \delta)}^{0(\Xi \Delta)} \eta_{\Lambda \Xi \eta_{\Sigma \Delta} \epsilon^{\alpha \gamma} \epsilon^{\beta \delta}} \\
& =-\left[T_{(a b)(\alpha \beta)}^{(\Lambda \Sigma)}-\frac{1}{n} \eta^{\Lambda \Sigma} \operatorname{Tr}_{\eta}\left(T_{(a b)(\alpha \beta)}\right)\right]\left[T_{(c d)(\gamma \delta)}^{(\Xi \Delta)}-\frac{1}{n} \eta^{\Xi \Delta} \operatorname{Tr}_{\eta}\left(T_{(c d)(\gamma \delta)}\right)\right] \eta_{\Lambda \Xi \eta_{\Sigma \Delta} \epsilon^{\alpha \gamma} \epsilon^{\beta \delta}} \\
& =-T_{(a b)(\alpha \beta)}^{(\Lambda \Sigma)} T_{(c d)(\gamma \delta)}^{(\Xi \Delta)} \eta_{\Lambda \Xi} \eta_{\Sigma \Delta} \epsilon^{\alpha \gamma} \epsilon^{\beta \delta}+\frac{1}{n} \operatorname{Tr}_{\eta}\left(T_{(a b)(\alpha \beta)}\right) \operatorname{Tr}_{\eta}\left(T_{(c d)(\gamma \delta)}\right) \epsilon^{\alpha \gamma} \epsilon^{\beta \delta} .
\end{aligned}
$$

It is convenient to introduce the following tensors (see also point 2 below):

$$
\begin{aligned}
& \mathcal{J}_{(a b)(c d)} \equiv-T_{(a b)(\alpha \beta)}^{(\Lambda \Sigma)} T_{(c d)(\gamma \delta)}^{(\Xi \Delta)} \eta_{\Lambda \Xi} \eta_{\Sigma \Delta} \epsilon^{\alpha \gamma} \epsilon^{\beta \delta} ; \\
& \mathbf{J}_{(a b)(c d)} \equiv \mathbf{T}_{(a b)(\alpha \beta)}^{(\Lambda \Sigma)} \mathbf{T}_{(c d)(\gamma \delta)}^{(\Xi \Delta)} \eta_{\Lambda \Xi} \eta_{\Sigma \Delta} \epsilon^{\alpha \gamma} \epsilon^{\beta \delta}=\frac{1}{n} \operatorname{Tr}_{\eta}\left(T_{(a b)(\alpha \beta)}\right) \operatorname{Tr}_{\eta}\left(T_{(c d)(\gamma \delta)}\right) \epsilon^{\alpha \gamma} \epsilon^{\beta \delta},
\end{aligned}
$$

such that eq. (2.23) can be rewritten as

$$
\mathcal{J}_{(a b)(c d)}^{0}=\mathcal{J}_{(a b)(c d)}+\mathbf{J}_{(a b)(c d)}
$$

Then, $\mathcal{J}_{(a b)(c d)}(2.24)$ can be $\mathrm{SL}_{h}(2, \mathbb{R})$-irreducibly decomposed as

$$
\underset{\mathbf{3} \times \mathbf{3}}{\mathcal{J}_{(a b)(c d)}}=-\operatorname{Tr}_{\eta}\left(\underset{\mathbf{5}_{s}^{0}+\mathbf{1}_{s}}{\left(T_{(a b)}\right.} T_{(c d))}\right)-\operatorname{Tr}_{\eta}\left(T_{[(a b)} T_{(c d)]}\right) .
$$

By making use of the cyclic property of $\operatorname{Tr}_{\eta}$ and of the distributivity of the sum with respect to it, one obtains

$$
\mathbf{3}_{a} \equiv-\operatorname{Tr}_{\eta}\left(T_{[(a b)} T_{(c d)]}\right)=0
$$


and therefore $\mathcal{J}_{(a b)(c d)}$ can be rewritten as

$$
\begin{aligned}
\mathcal{J}_{(a b)(c d)} & =-\operatorname{Tr}_{\eta}\left(T_{(a b)} T_{(c d)}\right)=-\operatorname{Tr}_{\eta}\left(T_{((a b)} T_{(c d))}\right) \\
& =-\frac{1}{3} \operatorname{Tr}_{\eta}\left(T_{(a b)} T_{(c d)}+T_{(a c)} T_{(b d)}+T_{(a d)} T_{(b c)}\right),
\end{aligned}
$$

with

$$
\begin{aligned}
\mathbf{5}_{s}^{0} & \equiv \mathcal{J}_{(a b c d)} \equiv \mathcal{J}_{(a b)(c d)}-\frac{1}{3}\left(\mathcal{X}-\frac{5}{2} \mathcal{W}^{2}\right) \epsilon_{a(c \mid} \epsilon_{b \mid d)} \\
\mathbf{1}_{s} & \equiv \mathcal{J}_{(a b)(c d)}-\mathcal{J}_{(a b c d)}=\frac{1}{3} \epsilon^{a^{\prime} c^{\prime}} \epsilon^{b^{\prime} d^{\prime}} \operatorname{Tr}_{\eta}\left(T_{\left(a^{\prime} b^{\prime}\right)} T_{\left(c^{\prime} d^{\prime}\right)}+T_{\left(a^{\prime} d^{\prime}\right)} T_{\left(b^{\prime} c^{\prime}\right)}\right) \epsilon_{a(c \mid} \epsilon_{b \mid d)} \\
& =\frac{1}{3}\left(\mathcal{X}-\frac{5}{2} \mathcal{W}^{2}\right) \epsilon_{a(c \mid} \epsilon_{b \mid d)}
\end{aligned}
$$

$\mathcal{X}$ is the order-4 "horizontal" invariant homogeneous polynomial defined by (4.13) of [9], which we report here, in the current notation (recall (2.12)-(2.14), as well as Footnote 3):

$$
\mathcal{X} \equiv-\operatorname{Tr}_{\eta}\left(T_{11}^{\prime} T_{22}^{\prime}\right)+\operatorname{Tr}_{\eta}\left(T_{12}^{\prime 2}\right)-\frac{1}{8} \operatorname{Tr}_{\eta}^{2}\left(T^{\prime \prime}\right) .
$$

In order to get an "horizontal" invariant polynomial homogeneous of order 4 in the fluxes, one has e.g. to $\epsilon$-trace both sides of (2.31), obtaining (as a consequence of the $\epsilon$-tracelessness of $\left.\mathcal{J}_{(a b c d)}(2.30)\right)$ :

$$
\epsilon^{a c} \epsilon^{b d} \mathcal{J}_{(a b)(c d)}=\frac{1}{3}\left(\mathcal{X}-\frac{5}{2} \mathcal{W}^{2}\right) \epsilon^{a c} \epsilon^{b d} \epsilon_{a(c \mid} \epsilon_{b \mid d)}=\mathcal{X}-\frac{5}{2} \mathcal{W}^{2}
$$

2.

$$
(\mathbf{3}, \mathbf{3}, \mathbf{1}) \times(\mathbf{3}, \mathbf{3}, \mathbf{1})=\left(\underset{G_{4} \text {-inv. }}{\mathbf{3}} \underset{\mathbf{3}}{\mathbf{3}}, \mathbf{1}\right)+\ldots
$$

There are no other sources of duality-invariant polynomials involving tensor products of $(\mathbf{3}, \mathbf{3}, \mathbf{1})$. The order- $4 G_{4}$-invariant polynomial from $(2.34)$ can reducibly be rewritten as the $\mathrm{SL}_{h}(2, \mathbb{R})$-bi-triplet $\mathbf{J}_{(a b)(c d)}$ defined by (2.25), which enjoys a decomposition analogous to the one of $\mathcal{J}_{(a b)(c d)}$.

3.

$$
(\mathbf{3}, \mathbf{1}, \mathbf{A d j}) \times(\mathbf{3}, \mathbf{1}, \mathbf{A d j})=\underset{G_{4} \text {-inv. }}{\mathbf{3}, \mathbf{1}, \mathbf{1})}+\ldots
$$

Since $\mathbf{1} \notin \mathbf{S}_{2} \times \mathbf{A d j}$, there are no other sources of duality-invariant polynomials involving tensor products of $(\mathbf{3}, \mathbf{1}, \mathbf{A d j})$. The order-4 $G_{4}$-invariant polynomial from (2.35) can reducibly be rewritten as the $\mathrm{SL}_{h}(2, \mathbb{R})$-bi-triplet

$$
T_{(a b)[\alpha \beta]}^{[\Lambda \Sigma]} T_{(c d)[\gamma \delta]}^{[\Xi \Delta]} \eta_{\Lambda \Xi \eta_{\Sigma \Delta}}
$$

However, without any loss of generality, one can instead consider (half of) the tensor product of the corresponding $\mathrm{SL}_{v}(2, \mathbb{R}) \epsilon$-traces (which is also the unique independent manifestly $\mathrm{SL}_{v}(2, \mathbb{R})$-invariant combination); by using the $\mathrm{SO}(2, n-2)$-matrix 
notation (2.13), one obtains the following $\mathrm{SL}_{h}(2, \mathbb{R})$-bi-triplet

$$
\begin{aligned}
I_{(a b)(c d)} & \equiv \frac{1}{2} T_{(a b)}^{[\Lambda \Sigma]} T_{(c d)}^{[\Xi \Delta]} \eta_{\Lambda \Xi \eta_{\Sigma \Delta}}=\frac{1}{2} \epsilon^{\alpha \beta} \epsilon^{\gamma \delta} T_{(a b)[\alpha \beta]}^{[\Lambda \Sigma]} T_{(c d)[\gamma \delta]}^{[\Xi \Delta]} \eta_{\Lambda \Xi \eta_{\Sigma \Delta}} \\
& =\frac{1}{2} T_{[\Lambda \Sigma](a b)} T_{(c d)}^{[\Lambda \Sigma]}=-\frac{1}{2} \operatorname{Tr}_{\eta}\left(T_{(a b)}^{\prime} T_{(c d)}^{\prime}\right) .
\end{aligned}
$$

Then, $I_{(a b)(c d)}$ can be $\mathrm{SL}_{h}(2, \mathbb{R})$-irreducibly decomposed as

$$
\left.\underset{\mathbf{3} \times \mathbf{3}}{I_{(a b)(c d)}}=-\frac{1}{2} \operatorname{Tr}_{\eta} \underset{\mathbf{5}_{s}^{0}+\mathbf{1}_{s}}{\left(T_{((a b)}^{\prime} T_{(c d))}^{\prime}\right)}\right)-\frac{1}{2} \operatorname{Tr}_{\eta}\left(\underset{\mathbf{3}_{a}}{\left(T_{(a b)}^{\prime}\right.} T_{(c d)]}^{\prime}\right) .
$$

Since

$$
\mathbf{3}_{a} \equiv-\frac{1}{2} \operatorname{Tr}_{\eta}\left(T_{[(a b)}^{\prime} T_{(c d)]}^{\prime}\right)=0,
$$

$I_{(a b)(c d)}$ can be rewritten as

$$
\begin{aligned}
I_{(a b)(c d)} & =-\frac{1}{2} \operatorname{Tr}_{\eta}\left(T_{(a b)}^{\prime} T_{(c d)}^{\prime}\right)=-\frac{1}{2} \operatorname{Tr}_{\eta}\left(T_{((a b)}^{\prime} T_{(c d))}^{\prime}\right) \\
& =-\frac{1}{6} \operatorname{Tr}_{\eta}\left(T_{(a b)}^{\prime} T_{(c d)}^{\prime}+T_{(a c)}^{\prime} T_{(b d)}^{\prime}+T_{(a d)}^{\prime} T_{(b c)}^{\prime}\right),
\end{aligned}
$$

with

$$
\begin{aligned}
\mathbf{5}_{s}^{0} & \equiv I_{(a b c d)} \equiv I_{(a b)(c d)}-\frac{1}{3}\left(\mathcal{X}+\frac{1}{2} \mathcal{W}^{2}\right) \epsilon_{a(c \mid} \epsilon_{b \mid d)} \\
\mathbf{1}_{s} & \equiv I_{(a b)(c d)}-I_{(a b c d)}=\frac{1}{3} \epsilon^{a^{\prime} c^{\prime}} \epsilon^{b^{\prime} d^{\prime}} \operatorname{Tr}_{\eta}\left(T_{\left(a^{\prime} b^{\prime}\right)}^{\prime} T_{\left(c^{\prime} d^{\prime}\right)}^{\prime}+T_{\left(a^{\prime} d^{\prime}\right)}^{\prime} T_{\left(b^{\prime} c^{\prime}\right)}^{\prime}\right) \epsilon_{a(c \mid} \epsilon_{b \mid d)} \\
& =\frac{1}{3}\left(\mathcal{X}+\frac{1}{2} \mathcal{W}^{2}\right) \epsilon_{a(c \mid} \epsilon_{b \mid d)} .
\end{aligned}
$$

$I_{(a b c d)}$ is the so-called Dixmier tensor [27] (or better its "two-centered analogue"), introduced in supergravity in [9-11,28], and generally related to the $\mathbb{K}$-tensor $\mathbb{K}_{(M N P Q)}$ of $G_{4}$ ([29]; see also [21] and refs. therein) as follows: ${ }^{4}$

$$
I_{(a b c d)} \equiv \frac{1}{2} \mathbb{K}_{M N P Q} \mathcal{Q}_{a}^{M} \mathcal{Q}_{b}^{N} \mathcal{Q}_{c}^{P} \mathcal{Q}_{d}^{Q} .
$$

In order to get an "horizontal" invariant polynomial homogeneous of order 4 in the fluxes, one has then to $\epsilon$-trace the unique $\epsilon$-traceful quantity out of (2.41)-(2.42), namely $\mathbf{1}_{s}$; by also recalling eq. (4.13) of [9], the following result (consequence of the $\epsilon$-tracelessness of $\left.I_{(a b c d)}(2.41)\right)$ is achieved:

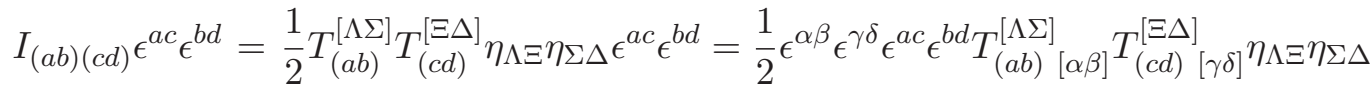

$$
\begin{aligned}
& =-\frac{1}{2} \epsilon^{a c} \epsilon^{b d} \operatorname{Tr}_{\eta}\left(T_{(a b)}^{\prime} T_{(c d)}^{\prime}\right)=\frac{1}{3}\left(\mathcal{X}+\frac{1}{2} \mathcal{W}^{2}\right) \epsilon^{a c} \epsilon^{b d} \epsilon_{a(c \mid} \epsilon_{b \mid d)} \\
& =\mathcal{X}+\frac{1}{2} \mathcal{W}^{2}=2\left(\mathbf{I}^{\prime}-\mathbf{I}^{\prime \prime}\right),
\end{aligned}
$$

\footnotetext{
${ }^{4}$ For a discussion of the differences between $\mathrm{CV}$ (i.e. reducible symmetric) and irreducible symmetric $D=4$ supergravity models, see [10] and [11].
} 
where $\mathbf{I}^{\prime}$ and $\mathbf{I}^{\prime \prime}$ are duality-invariant order-4 polynomials respectively defined by (3.12) and (3.13) of [9], which we report here in current notation (recall Footnote 3):

$$
\left.\begin{array}{l}
\mathbf{I}^{\prime} \equiv-\frac{1}{2} \operatorname{Tr}_{\eta}\left(T_{11}^{\prime} T_{22}^{\prime}\right) ; \\
\mathbf{I}^{\prime \prime} \equiv-\frac{1}{2} \operatorname{Tr}_{\eta}\left(T_{12}^{\prime 2}\right) ;
\end{array}\right\} \Rightarrow \mathbf{I}^{\prime}-\mathbf{I}^{\prime \prime}=\frac{1}{2}\left[\operatorname{Tr}_{\eta}\left(T_{12}^{\prime 2}\right)-\operatorname{Tr}_{\eta}\left(T_{11}^{\prime} T_{22}^{\prime}\right)\right]
$$

By virtue of (2.15) and (2.32), it also holds that (cfr. eq. (4.13) of [9]):

$$
\mathbf{I}^{\prime}-\mathbf{I}^{\prime \prime}=\frac{1}{2}\left(\mathcal{X}+\frac{1}{2} \mathcal{W}^{2}\right) .
$$

Furthermore, one can derive a simple identity relating $I_{(a b)(c d)}(2.37)$ and $\mathcal{J}_{(a b)(c d)}(2.24)$ :

$$
\mathcal{J}_{(a b)(c d)}=I_{(a b)(c d)}-\mathcal{W}^{2} \epsilon_{a(c \mid} \epsilon_{b \mid d)},
$$

in turn implying

$$
\mathcal{J}_{(a b c d)}=I_{(a b c d)}
$$

4.

$$
(\mathbf{1}, \mathbf{3}, \mathbf{A d j}) \times(\mathbf{1}, \mathbf{3}, \mathbf{A d j})=\frac{(\mathbf{1}, \mathbf{1}, \mathbf{1})}{\left[\mathrm{SL}_{h}(2, \mathbb{R}) \times G_{4}\right] \text {-inv. }}+\ldots
$$

There are no other sources of duality- (nor "horizontal"-)invariant polynomials involving tensor products of $(\mathbf{1}, \mathbf{3}, \mathbf{A d j})$. The order-4 "horizontal" invariant polynomial from (2.49) can irreducibly be written as

$$
T_{[a b](\alpha \beta)}^{[\Lambda \Sigma]} T_{[c d](\beta \gamma)}^{[\Xi \Delta]} \eta_{\Lambda \Xi} \eta_{\Sigma \Delta} \epsilon^{\alpha \gamma} \epsilon^{\beta \delta} .
$$

However, without any loss of generality, one can instead consider (half of) the tensor product of the corresponding $\mathrm{SL}_{h}(2, \mathbb{R}) \epsilon$-traces (which is also the unique independent manifestly $\mathrm{SL}_{h}(2, \mathbb{R})$-invariant combination), obtaining

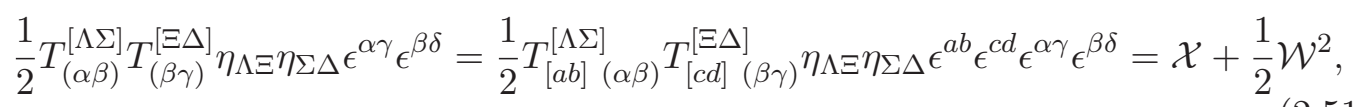

consistently matching the result (2.44), because eqs. (2.44) and (2.51) actually share the same left-hand side.

5.

$$
\left(\mathbf{1}, \mathbf{1}, \mathbf{S}_{0}\right) \times\left(\mathbf{1}, \mathbf{1}, \mathbf{S}_{0}\right)=\underset{\left[\mathrm{SL}_{h}(2, \mathbb{R}) \times G_{4}\right] \text {-inv. }}{(\mathbf{1}, \mathbf{1})}+\ldots
$$

Since $\mathbf{1} \notin \mathbf{S}_{2} \times \mathbf{A d} \mathbf{j}$, there are no other sources of duality- (nor "horizontal"-)invariant polynomials involving tensor products of $\left(\mathbf{1}, \mathbf{1}, \mathbf{S}_{0}\right)$. The order-4 "horizontal" invariant polynomial from (2.52) can irreducibly be written as

$$
\begin{aligned}
T_{[a b][\alpha \beta]}^{0(\Lambda \Sigma)} T_{[a b][\alpha \beta]}^{0(\Xi \Delta)} \eta_{\Lambda \Xi \eta_{\Sigma \Delta}=} & {\left[T_{[a b][\alpha \beta]}^{(\Lambda \Sigma)}-\frac{1}{n} \eta^{\Lambda \Sigma} \operatorname{Tr}_{\eta}\left(T_{[a b][\alpha \beta]}^{\prime \prime}\right)\right] . } \\
& \cdot\left[T_{[c d][\gamma \delta]}^{(\Xi \Delta)}-\frac{1}{n} \eta^{\Lambda \Sigma} \operatorname{Tr}_{\eta}\left(T_{[c d][\gamma \delta]}^{\prime \prime}\right)\right] \eta_{\Lambda \Xi \eta_{\Sigma \Delta}},
\end{aligned}
$$


where the $\mathrm{SO}(2, n-2)$-matrix notation (2.14) has been recalled. However, without any loss of generality, one can instead consider the tensor product of the corresponding $\mathrm{SL}_{h}(2, \mathbb{R})$ and $\mathrm{SL}_{v}(2, \mathbb{R}) \epsilon$-traces (which is also the unique independent manifestly $S O_{h}^{v}(2,2)$-invariant combination), obtaining

$$
\begin{aligned}
& T^{0}{ }^{(\Lambda \Sigma)} T^{0(\Xi \Delta)} \eta_{\Lambda \Xi} \eta_{\Sigma \Delta} \equiv \epsilon^{a b} \epsilon^{\alpha \beta} \epsilon^{c d} \epsilon^{\gamma \delta} T_{[a b][\alpha \beta]}^{0(\Lambda \Sigma)} T_{[c d][\gamma \delta]}^{0(\Xi \Delta)} \eta_{\Lambda \Xi \eta_{\Sigma \Delta}} \\
&=\epsilon^{a b} \epsilon^{\alpha \beta} \epsilon^{c d} \epsilon^{\gamma \delta}\left[T_{[a b][\alpha \beta]}^{(\Lambda \Sigma)}-\frac{1}{n} \eta^{\Lambda \Sigma} \operatorname{Tr}_{\eta}\left(T_{[a b][\alpha \beta]}^{\prime \prime}\right)\right] \\
& \cdot\left[T_{[c d][\gamma \delta]}^{(\Xi \Delta)}-\frac{1}{n} \eta^{\Xi \Delta} \operatorname{Tr}_{\eta}\left(T_{[c d][\gamma \delta]}^{\prime \prime}\right)\right] \eta_{\Lambda \Xi \eta_{\Sigma \Delta}} \\
&= \epsilon^{a b} \epsilon^{\alpha \beta} \epsilon^{c d} \epsilon^{\gamma \delta} T_{[a b][\alpha \beta]}^{(\Lambda \Sigma)} T_{[c d][\gamma \delta]}^{(\Xi \Delta)} \eta_{\Lambda \Xi \eta_{\Sigma \Delta} \Delta} \\
&-\frac{1}{n} \epsilon^{a b} \epsilon^{\alpha \beta} \epsilon^{c d} \epsilon^{\gamma \delta} \operatorname{Tr}_{\eta}\left(T_{[a b][\alpha \beta]}^{\prime \prime}\right) \operatorname{Tr}_{\eta}\left(T_{[c d][\gamma \delta]}^{\prime \prime}\right) \\
& \equiv T^{(\Lambda \Sigma)} T^{(\Xi \Delta)} \eta_{\Lambda \Xi} \eta_{\Sigma \Delta}-\frac{1}{n} \operatorname{Tr}_{\eta}\left(T^{\prime \prime}\right) \operatorname{Tr}_{\eta}\left(T^{\prime \prime}\right) .
\end{aligned}
$$

Observing that the definition (2.15) can be rewritten as

$$
\operatorname{Tr}_{\eta}\left(T^{\prime \prime}\right)=2 \mathcal{W}
$$

definitions (2.6) imply that

$$
T^{0(\Lambda \Sigma)} \equiv T^{(\Lambda \Sigma)}-\frac{2}{n} \eta^{\Lambda \Sigma} \mathcal{W}
$$

On the other hand, an explicit computation yields

$$
T^{(\Lambda \Sigma)} T^{(\Xi \Delta)} \eta_{\Lambda \Xi \eta_{\Sigma \Delta}}=-2\left(2 \mathcal{X}-\mathcal{W}^{2}\right)
$$

Therefore, by inserting (2.55)-(2.57) into (2.54), the following expression of the corresponding order-4 "horizontal" invariant polynomial is achieved:

$$
T^{0(\Lambda \Sigma)} T^{0\left({ }^{(\Xi)}\right)} \eta_{\Lambda \Xi \eta_{\Sigma \Delta}}=-4 \mathcal{X}+\left(2-\frac{4}{n}\right) \mathcal{W}^{2} .
$$

Note that, from (1.1) and observations below, the coefficient of $\mathcal{W}^{2}$ in (2.58) is strictly positive in all CV models.

6.

$$
(\mathbf{1}, \mathbf{1}, \mathbf{1}) \times(\mathbf{1}, \mathbf{1}, \mathbf{1})=\frac{(\mathbf{1}, \mathbf{1}, \mathbf{1})}{\left[\mathrm{SL}_{h}(2, \mathbb{R}) \times G_{4}\right] \text {-inv. }}
$$

By recalling (2.6), the order-4 "horizontal" invariant polynomial from (2.59) can irreducibly be written as

$$
\mathbf{T}_{[a b][\alpha \beta]}^{(\Lambda \Sigma)} \mathbf{T}_{[c d][\gamma \delta]}^{(\Xi \Delta)} \eta_{\Lambda \Xi} \eta_{\Sigma \Delta} \equiv \frac{1}{n} \operatorname{Tr}_{\eta}\left(T_{[a b][\alpha \beta]}^{\prime \prime}\right) \operatorname{Tr}_{\eta}\left(T_{[c d][\gamma \delta]}^{\prime \prime}\right) .
$$


However, without any loss of generality, one can instead consider the tensor product of the corresponding $\mathrm{SL}_{h}(2, \mathbb{R})$ and $\mathrm{SL}_{v}(2, \mathbb{R}) \epsilon$-traces (once again, the unique independent manifestly $S O_{h}^{v}(2,2)$-invariant combination), obtaining

$$
\begin{aligned}
\mathbf{T}^{(\Lambda \Sigma)} \mathbf{T}^{(\Xi \Delta)} \eta_{\Lambda \Xi \eta_{\Sigma \Delta}} & \equiv \epsilon^{a b} \epsilon^{\alpha \beta} \epsilon^{c d} \epsilon^{\gamma \delta} \mathbf{T}_{[a b][\alpha \beta]}^{(\Lambda \Sigma)} \mathbf{T}_{[c d][\gamma \delta]}^{(\Xi \Delta)} \eta_{\Lambda \Xi \eta_{\Sigma \Delta}} \\
& =\frac{1}{n} \epsilon^{a b} \epsilon^{\alpha \beta} \epsilon^{c d} \epsilon^{\gamma \delta} \operatorname{Tr}_{\eta}\left(T_{[a b][\alpha \beta]}^{\prime \prime}\right) \operatorname{Tr}_{\eta}\left(T_{[c d][\gamma \delta]}^{\prime \prime}\right) \\
& =\frac{1}{n} \operatorname{Tr}_{\eta}\left(T^{\prime \prime}\right) \operatorname{Tr}_{\eta}\left(T^{\prime \prime}\right)=\frac{4}{n} \mathcal{W}^{2},
\end{aligned}
$$

where eq. (2.55) was used.

\subsubsection{Summary}

The above analysis completes, at order 4 in the fluxes, the treatment given in [9] and [11].

Besides $\mathcal{W}^{2}$ and $\mathcal{X}$, no other "horizontal" invariant homogeneous polynomials of order 4 in the BH fluxes $\mathcal{Q}_{1 \alpha}^{\Lambda}$ and $\mathcal{Q}_{2 \alpha}^{\Lambda}$ can be introduced.

Concerning duality-invariant homogeneous polynomials of order 4, the Dixmier tensor $I_{(a b c d)}[27]$, sitting in the spin $s=2$ irrep. 5 of the "horizontal" symmetry $\operatorname{SL}_{h}(2, \mathbb{R})$, generally defined by $(2.43)$ and present in the analysis of $[9,10]$, is (due to $(2.48)$ ) the unique algebraically independent duality-invariant tensor sitting in an irrep. of $\mathrm{SL}_{h}(2, \mathbb{R})$ itself. Other duality-invariant tensors of mixed "horizontal" symmetry, such as $I_{(a b)(c d)}(2.37)$ and $\mathcal{J}_{(a b)(c d)}(2.24)$ (related by $\left.(2.47)\right)$ can be introduced, but they do not sit in "horizontal" irreps..

\section{$2.3 \quad$ Order 6}

Since there is no duality- nor "horizontal" - invariant polynomial structure at order 5, we proceed to analyze the order 6 in the fluxes of the 2-centered BH solution in the framework under consideration. By exploiting the associativity of the (ir)reps.' tensor product, in each of the $\mathrm{SL}_{h}(2, \mathbb{R})$ - and $\mathrm{SL}_{v}(2, \mathbb{R})$ - sectors one gets

$$
\mathbf{2} \times \mathbf{2} \times \mathbf{2} \times \mathbf{2} \times \mathbf{2} \times \mathbf{2}=(\mathbf{1}+\mathbf{3}+\mathbf{5}+2 \cdot \mathbf{3}+\mathbf{1}) \times(\mathbf{3}+\mathbf{1})=5 \cdot \mathbf{1}+9 \cdot \mathbf{3}+5 \cdot \mathbf{5}+\mathbf{7} .
$$

On the other hand, in the $\mathrm{SO}(2, n-2)$ sector the sources of singlets list as follows:

$$
\begin{aligned}
& \text { Adj } \times \text { Adj } \times \text { Adj }=1+\ldots ; \\
& \operatorname{Adj} \times \operatorname{Adj} \times S_{0}=\mathbf{1}+\ldots ; \\
& \operatorname{Adj} \times \operatorname{Adj} \times \mathbf{1}=\mathbf{1}+\ldots ; \\
& \mathbf{S}_{0} \times \mathbf{S}_{0} \times \mathbf{A d j}=\mathbf{1}+\ldots ; \\
& \mathbf{S}_{0} \times \mathbf{S}_{0} \times \mathbf{S}_{0}=\mathbf{1}+\ldots ; \\
& \mathbf{S}_{0} \times \mathbf{S}_{0} \times \mathbf{1}=\mathbf{1}+\ldots ; \\
& \mathbf{1} \times \mathbf{1} \times \mathbf{1}=\mathbf{1}
\end{aligned}
$$

Thus, the "horizontal" invariant homogeneous polynomials at order 6 in the fluxes arise as singlets $(\mathbf{1}, \mathbf{1}, \mathbf{1})$ among other representations from the following tensor products (in determining the corresponding tensor structure, we will disregard the irreducible splitting (2.2), irrelevant for our purposes): 
1.

$$
\begin{aligned}
& \left(\mathbf{3}, \mathbf{3}, \mathbf{S}_{0}\right) \times\left(\mathbf{3}, \mathbf{3}, \mathbf{S}_{0}\right) \times\left(\mathbf{3}, \mathbf{3}, \mathbf{S}_{0}\right) \\
& \left(\mathbf{3}, \mathbf{3}, \mathbf{S}_{0}\right) \times\left(\mathbf{3}, \mathbf{3}, \mathbf{S}_{0}\right) \times(\mathbf{3}, \mathbf{3}, \mathbf{1}) \\
& (\mathbf{3}, \mathbf{3}, \mathbf{1}) \times(\mathbf{3}, \mathbf{3}, \mathbf{1}) \times(\mathbf{3}, \mathbf{3}, \mathbf{1})
\end{aligned}
$$

whose singlets correspond to the following 5 "horizontal" invariant homogeneous polynomial of order 6 :

$$
T_{(a b)(\alpha \beta)}^{(\Lambda \Sigma)} T_{(c d)(\gamma \delta)}^{(\Xi \Delta)} T_{(e f)(\eta \lambda)}^{(\Gamma \Pi)} \epsilon^{a f} \epsilon^{b c} \epsilon^{d e} \epsilon^{\alpha \lambda} \epsilon^{\beta \gamma} \epsilon^{\delta \eta} \eta_{\Lambda \Pi} \eta_{\Sigma \Xi} \eta_{\Delta \Gamma}=24 \mathbf{I}_{6}-24 \mathcal{W} \mathcal{X}+12 \mathcal{W}^{3},
$$

where $\mathbf{I}_{6}$ is the order-6 "horizontal" invariant polynomial defined by (3.16) of [9], which we report here in current notation (recall Footnote 3 and notation (2.13)):

$$
\mathbf{I}_{6} \equiv-\operatorname{Tr}_{\eta}\left(T_{11}^{\prime} T_{22}^{\prime} T_{12}^{\prime}\right) \text {. }
$$

2.

$$
\begin{aligned}
& \left(3,3, \mathbf{S}_{0}\right) \times\left(\mathbf{3}, \mathbf{3}, \mathbf{S}_{0}\right) \times\left(\mathbf{1}, \mathbf{1}, \mathbf{S}_{0}\right) \\
& (\mathbf{3}, \mathbf{3}, \mathbf{1}) \times(\mathbf{3}, \mathbf{3}, \mathbf{1}) \times(\mathbf{1}, \mathbf{1}, \mathbf{1}) \\
& \left(\mathbf{3}, \mathbf{3}, \mathbf{S}_{0}\right) \times(\mathbf{3}, \mathbf{3}, \mathbf{1}) \times\left(\mathbf{1}, \mathbf{1}, \mathbf{S}_{0}\right) \\
& \left(\mathbf{3}, \mathbf{3}, \mathbf{S}_{0}\right) \times\left(\mathbf{3}, \mathbf{3}, \mathbf{S}_{0}\right) \times(\mathbf{1}, \mathbf{1}, \mathbf{1})
\end{aligned}
$$

whose singlets correspond to the following "horizontal" invariant:

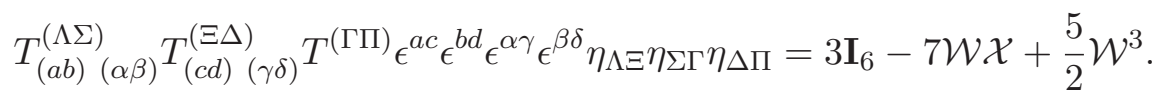

3.

$$
\begin{aligned}
& \left(\mathbf{3}, \mathbf{3}, \mathbf{S}_{0}\right) \times(\mathbf{3}, \mathbf{1}, \mathbf{A d j}) \times(\mathbf{1}, \mathbf{3}, \mathbf{A d j}) \\
& (\mathbf{3}, \mathbf{3}, \mathbf{1}) \times(\mathbf{3}, \mathbf{1}, \mathbf{A d j}) \times(\mathbf{1}, \mathbf{3}, \mathbf{A d j}),
\end{aligned}
$$

whose singlets correspond to the following "horizontal" invariant:

$$
T_{(a b)(\alpha \beta)}^{(\Lambda \Sigma)} T_{(c d)}^{[\Xi \Delta]} T_{(\eta \lambda)}^{[\Gamma \Pi]} \epsilon^{a c} \epsilon^{b d} \epsilon^{\alpha \eta} \epsilon^{\beta \lambda} \eta_{\Lambda \Pi} \eta_{\Sigma \Xi} \eta_{\Delta \Gamma}=3 \mathbf{I}_{6}+\mathcal{W} \mathcal{X}+\frac{1}{2} \mathcal{W}^{3} .
$$

4.

$$
(\mathbf{3}, \mathbf{1}, \operatorname{Adj}) \times(\mathbf{3}, \mathbf{1}, \operatorname{Adj}) \times(\mathbf{3}, \mathbf{1}, \mathbf{A d j}),
$$

whose singlet corresponds to the following "horizontal" invariant (recall definition $(2.66))$ :

$$
T_{(a b)}^{[\Lambda \Sigma]} T_{(c d)}^{[\Xi \Delta]} T_{(e f)}^{[\Gamma \Pi]} \epsilon^{a f} \epsilon^{b c} \epsilon^{d e} \eta_{\Lambda \Pi} \eta_{\Sigma \Xi} \eta_{\Delta \Gamma}=6 \mathbf{I}_{6} .
$$

5.

$$
\begin{aligned}
& (\mathbf{3}, \mathbf{1}, \mathbf{A d j}) \times(\mathbf{3}, \mathbf{1}, \mathbf{A d j}) \times\left(\mathbf{1}, \mathbf{1}, \mathbf{S}_{0}\right) ; \\
& (\mathbf{3}, \mathbf{1}, \text { Adj }) \times(\mathbf{3}, \mathbf{1}, \mathbf{A d j}) \times(\mathbf{1}, \mathbf{1}, \mathbf{1}),
\end{aligned}
$$

whose singlets correspond to the following "horizontal" invariant:

$$
T_{(a b)}^{[\Lambda \Sigma]} T_{(c d)}^{[\Xi \Delta]} T^{(\Gamma \Pi)} \epsilon^{a c} \epsilon^{b d} \epsilon^{\alpha \eta} \epsilon^{\beta \lambda} \eta_{\Lambda \Pi \eta_{\Sigma \Xi} \eta_{\Delta \Gamma}}=6 \mathbf{I}_{6}-2 \mathcal{W} \mathcal{X}-\mathcal{W}^{3} .
$$

\footnotetext{
${ }^{5}$ As in all cases, the reported index-contraction structure is the unique independent one (possibly taking into account the splitting (2.2), as well).
} 
6.

$$
(\mathbf{1}, \mathbf{3}, \mathbf{A d j}) \times(\mathbf{1}, \mathbf{3}, \mathbf{A d j}) \times(\mathbf{1}, \mathbf{3}, \mathbf{A d j}),
$$

whose singlet corresponds to the "horizontal" invariant (2.72):

$$
T_{(\alpha \beta)}^{[\Lambda \Sigma]} T_{(\gamma \delta)}^{[\Xi \Delta]} T_{(\eta \lambda)}^{[\Gamma \Pi]} \epsilon^{\alpha \lambda} \epsilon^{\beta \gamma} \epsilon^{\delta \eta} \eta_{\Lambda \Pi} \eta_{\Sigma \Xi} \eta_{\Delta \Gamma}=6 \mathbf{I}_{6},
$$

which makes the "horizontal" invariance of definition (2.66) manifest.

7.

$$
\begin{aligned}
& (\mathbf{1}, \mathbf{3}, \mathbf{A d j}) \times(\mathbf{1}, \mathbf{3}, \mathbf{A d j}) \times\left(\mathbf{1}, \mathbf{1}, \mathbf{S}_{0}\right) \\
& (\mathbf{1}, \mathbf{3}, \mathbf{A d j}) \times(\mathbf{1}, \mathbf{3}, \mathbf{A d j}) \times(\mathbf{1}, \mathbf{1}, \mathbf{1})
\end{aligned}
$$

whose singlets correspond to the "horizontal" invariant (2.74):

$$
T_{(\alpha \beta)}^{[\Lambda \Sigma]} T_{(\gamma \delta)}^{[\Xi \Delta]} T^{(\Gamma \Pi)} \epsilon^{\alpha \gamma} \epsilon^{\beta \delta} \eta_{\Lambda \Pi} \eta_{\Sigma \Xi} \eta_{\Delta \Gamma}=6 \mathbf{I}_{6}-2 \mathcal{W} \mathcal{X}-\mathcal{W}^{3} .
$$

8.

$$
\begin{aligned}
& \left(1,1, \mathbf{S}_{0}\right) \times\left(\mathbf{1}, \mathbf{1}, \mathbf{S}_{0}\right) \times\left(\mathbf{1}, \mathbf{1}, \mathbf{S}_{0}\right) \\
& \left(\mathbf{1}, \mathbf{1}, \mathbf{S}_{0}\right) \times\left(\mathbf{1}, \mathbf{1}, \mathbf{S}_{0}\right) \times(\mathbf{1}, \mathbf{1}, \mathbf{1}) \\
& (\mathbf{1}, \mathbf{1}, \mathbf{1}) \times(\mathbf{1}, \mathbf{1}, \mathbf{1}) \times(\mathbf{1}, \mathbf{1}, \mathbf{1})
\end{aligned}
$$

whose singlets correspond to the following "horizontal" invariant:

$$
T^{(\Lambda \Sigma)} T^{(\Xi \Delta)} T^{(\Gamma \Pi)} \eta_{\Lambda \Pi} \eta_{\Sigma \Xi} \eta_{\Delta \Gamma}=12 \mathbf{I}_{6}-12 \mathcal{W} \mathcal{X}+2 \mathcal{W}^{3} .
$$

9.

$$
\left(\mathbf{3}, \mathbf{3}, \mathbf{S}_{0}\right) \times\left(\mathbf{3}, \mathbf{3}, \mathbf{S}_{0}\right) \times(\mathbf{3}, \mathbf{1}, \mathbf{A d} \mathbf{j}),
$$

whose singlet corresponds to the following "horizontal" invariant:

$$
T_{(a b)(\alpha \beta)}^{(\Lambda \Sigma)} T_{(c d)(\gamma \delta)}^{(\Xi \Delta)} T_{(e f)}^{[\Gamma \Pi]} \epsilon^{\alpha f} \epsilon^{b c} \epsilon^{d e} \epsilon^{\alpha \gamma} \epsilon^{\beta \delta} \eta_{\Lambda \Pi} \eta_{\Sigma \Xi} \eta_{\Delta \Gamma}=3 \mathbf{I}_{6}-2 \mathcal{W} \mathcal{X}-\mathcal{W}^{3} .
$$

10.

$$
\left(\mathbf{3}, \mathbf{3}, \mathbf{S}_{0}\right) \times\left(\mathbf{3}, \mathbf{3}, \mathbf{S}_{0}\right) \times(\mathbf{1}, \mathbf{3}, \mathbf{A d j}),
$$

whose singlet corresponds to the "horizontal" invariant (2.82):

$$
T_{(a b)(\alpha \beta)}^{(\Lambda \Sigma)} T_{(c d)(\gamma \delta)}^{(\Xi \Delta)} T_{(\eta \lambda)}^{[\Gamma \Pi]} \epsilon^{\alpha c} \epsilon^{b d} \epsilon^{\alpha \lambda} \epsilon^{\beta \gamma} \epsilon^{\delta \eta} \eta_{\Lambda \Pi} \eta_{\Sigma \Xi} \eta_{\Delta \Gamma}=3 \mathbf{I}_{6}-2 \mathcal{W} \mathcal{X}-\mathcal{W}^{3} .
$$

\subsubsection{Summary}

The above analysis completes, at order 6 in the fluxes, the treatment given in [9] and [11].

Besides $\mathcal{W}^{3}$ and $\mathcal{W} \mathcal{X}$, also the "horizontal" invariant $\mathbf{I}_{6}$ (2.66) can be introduced. Concerning this, it is worth recalling here that two "horizontal" invariant order-6 homogeneous polynomials can be naturally introduced in CV models:

- the $\mathbf{I}_{6}$ defined by (2.66) above [9]; 
- the $\mathbf{I}_{6}^{\prime}$ given by (3.11) and (3.24) of [10], whose manifestly "horizontal"-invariant formulation in the CV symplectic frame [16] reads

$$
\begin{aligned}
\mathbf{I}_{6}^{\prime} & \equiv \frac{1}{2} \mathbb{C}_{\alpha \beta}^{\Lambda \Sigma} \widetilde{\mathcal{Q}}_{\Lambda \mid a b c}^{\alpha} \widetilde{\mathcal{Q}}_{\Sigma \mid d e f}^{\beta} \epsilon^{a d} \epsilon^{b e} \epsilon^{c f} \\
\widetilde{\mathcal{Q}}_{\Lambda \mid a b c}^{\alpha} & \equiv \frac{1}{2} \mathbb{K}_{\Lambda \Sigma \Xi \Delta}^{\alpha \beta \gamma \delta} \mathcal{Q}_{a \beta}^{\Sigma} \mathcal{Q}_{b \gamma}^{\Xi} \mathcal{Q}_{c \delta}^{\Delta},
\end{aligned}
$$

where, the symplectic-invariant $\mathbb{C}$-structure reads (consistent with the $\mathrm{CV}$ splitting (1.5))

$$
\mathbb{C}_{\alpha \beta}^{\Lambda \Sigma}=\left(\begin{array}{cc}
0^{\Lambda \Sigma} & -\delta_{\Sigma}^{\Lambda} \\
\delta_{\Lambda}^{\Sigma} & 0_{\Lambda \Sigma}
\end{array}\right)
$$

The general ${ }^{6}$ relation between $\mathbf{I}_{6}$ and $\mathbf{I}_{6}^{\prime}$ is discussed in section 3 of [11]; in CV models, such a relation is given by (also recall (2.44) and (2.45))

$$
\mathbf{I}_{6}=\mathbf{I}_{6}^{\prime}-\frac{1}{6} \mathcal{X} \mathcal{W}-\frac{1}{12} \mathcal{W}^{3}=\mathbf{I}_{6}^{\prime}-\frac{1}{3}\|\mathbb{T}\|^{2} \mathcal{W}
$$

which, besides (2.76), provides a manifestly "horizontal"-invariant expression of $\mathbf{I}_{6}$ in the CV symplectic frame.

\section{Invariants of pseudo-unitary $U$-duality}

We now discuss the "horizontal" symmetry of $D=4$ supergravity models with $U$-duality group $G_{4}$ given by the pseudo-unitary group $\mathrm{U}(r, s)$ for some $r$ and $s$. Confining ourselves to theories with symmetric scalar manifolds, these supergravity theories are:

- the $\mathcal{N}=2$ minimally coupled Maxwell-Einstein theory [30, 31] $(r=1)$, with scalar manifold

$$
M_{\mathcal{N}=2}=\frac{\mathrm{U}(1, s)}{\mathrm{U}(1) \times \mathrm{U}(s)} \sim \frac{\mathrm{SU}(1, s)}{\mathrm{SU}(s) \times \mathrm{U}(1)} \equiv \mathbb{C P}^{s}
$$

and vector 2 -form field strengths sitting in the (complex) fundamental irrep. $\mathbf{s}+\mathbf{1}$ of $G_{4}=\mathrm{U}(1, s)$;

- the $\mathcal{N}=3$ matter-coupled theory [32] $(r=3)$, with scalar manifold

$$
M_{\mathcal{N}=3}=\frac{\mathrm{U}(3, s)}{\mathrm{U}(1) \times S(\mathrm{U}(3) \times \mathrm{U}(s))} \sim \frac{\mathrm{SU}(3, s)}{\mathrm{SU}(3) \times \mathrm{SU}(s) \times \mathrm{U}(1)},
$$

and vector 2 -form field strengths sitting in the (complex) fundamental irrep. $\mathbf{s}+\mathbf{3}$ of $G_{4}=\mathrm{U}(3, s)$.

${ }^{6}$ As discussed in [10] and in [11], an important difference between CV models and those $D=4$ symmetric models with simple $U$-duality groups (named irreducible symmetric models in such refs.) is that in these latter $\mathcal{X}$ vanishes identically (due to the holding of eq. (3.7) of [10]). 
It is here worth recalling that $\mathcal{N}=2$ supergravity minimally coupled to 3 vector multiplets is "bosonic twin" to (i.e. shares the very same bosonic sector of) $\mathcal{N}=3$ supergravity coupled to 1 vector multiplet [31,33] (for a discussion of split flows and marginal stability in extended $D=4$ supergravities, see e.g. [34]).

As observed in [26] (in which the split attractor flow and marginal stability features of theories (3.1) were investigated), the presence of an "extra" $U(1)$ symmetry, acting only on the complex(ified) flux vector $\mathcal{Q}$ but not on the (complex) scalar fields (see e.g. (2.35)(2.36) of [26]) is due to the fact that such theories are the only ones in which the "pure" theory limit (corresponding to the case in which only the graviton multiplet present) can be obtained by simply setting to zero the number $s$ of matter (Abelian vector) multiplets. As such, the "extra" U(1) global factor ${ }^{7}$ (which is not a global isometry of the scalar manifold) is nothing but the $\mathrm{U}(1)$ symmetry gauged by the complex scalars, which becomes global in their absence [35] (recall that the $\mathcal{N}=2$ and $\mathcal{N}=3$ graviton multiplets do not contain scalar fields). Moreover, in the $\mathcal{N}=2$ case, such a $\mathrm{U}(1)$ can also be interpreted as the symmetry of the graviphotonic electro-magnetic system.

\section{1 $\quad \mathrm{SL}(p, \mathbb{C}) \times \mathrm{U}(r, s)$}

Before dealing with the actual "horizontal" symmetry of these theories, it is instructive to consider the group

$$
\mathrm{SL}(p, \mathbb{C}) \times \mathrm{U}(r, s),
$$

and the orbits of complex vectors $\mathcal{V}_{i}^{A}(A=1, \ldots, r+s, i=1, \ldots, p)$ in the complex bifundamental irrep. $(\mathbf{p}, \mathbf{r}+\mathbf{s})$ of $\mathrm{SL}(p, \mathbb{C}) \times \mathrm{U}(r, s)$. This treatment can be considered the "complexified version" of the treatment given in the second part of section 4 of [10], and also the results will be analogous.

There are only $p^{2}$ algebraically-independent $\mathrm{U}(r, s)$-invariant homogeneous polynomials, all of order 2 in the fluxes, given by

$$
\mathbf{U}_{i \bar{j}} \equiv \mathcal{V}_{i}^{A} \overline{\mathcal{V}}_{\bar{j}}^{\bar{B}} \eta_{A \bar{B}} \equiv \mathcal{V}_{i} \cdot \overline{\mathcal{V}}_{\bar{j}}
$$

where "." denotes the scalar product determined by the pseudo-Euclidean metric $\eta_{A \bar{B}}$ of $\mathrm{U}(r, s)$. By respectively denoting with $\mathbf{I}_{p}$ and $\mathfrak{G}_{p}$ the dimension of a complete basis of $\mathrm{U}(r, s)$-invariant polynomials and the orbit of the irrep. $\mathbf{r}+\mathbf{s}$ of $\mathrm{U}(r, s)$, the counting

$$
\mathbf{I}_{p}=p^{2}
$$

is consistent with the general counting rule $[9,10]$ :

$$
\mathbf{I}_{p}=2(r+s) p-\operatorname{dim}_{\mathbb{R}}\left(\mathfrak{G}_{p}\right),
$$

because $\mathfrak{G}_{p}$ generally is a suitable non-compact form of the compact coset

$$
\mathfrak{G}_{p, \text { compact }}=\frac{\mathrm{U}(r+s)}{\mathrm{U}(r+s-p)}, \operatorname{dim}_{\mathbb{R}}=2(r+s) p-p^{2} .
$$

\footnotetext{
${ }^{7}$ At least for $p=2$, the relevance of the "extra" $\mathrm{U}(1)$ factor for the counting of $\mathrm{U}(r, s)$-invariant polynomials has been discussed at the end of page 5 of [26].
} 
On the other hand, out of the $p^{2}$ order-2 $\mathrm{U}(r, s)$-invariant polynomials $\mathbf{U}_{i \bar{j}}(3.4)$, one can construct a unique algebraically-independent $[\mathrm{SL}(p, \mathbb{C}) \times \mathrm{U}(r, s)]$ - invariant homogeneous polynomial, of order $2 p$, defined as

$$
\operatorname{det} \widehat{\mathbf{G}}=\mathcal{V}_{i_{1} \bar{j}_{1}} \mathcal{V}_{i_{2} \bar{j}_{2}} \ldots \mathcal{V}_{i_{p} \bar{j}_{p}} \epsilon^{i_{1} i_{2} \ldots i_{p}} \epsilon^{\bar{j}_{1} \bar{j}_{2} \ldots \bar{j}_{p}}
$$

The notation "det $\widehat{\mathbf{G}}$ " indicates the fact that the "horizontal"-invariant polynomial (3.8) is the determinant of the Hermitian-analogue of the Gramian matrix G introduced in (8.4)(8.5) of [9] (see also the treatment of section 8 therein). In the 2 -centered case $(p=2),(3.8)$ reduces to

$$
\operatorname{det} \widehat{\mathbf{G}}=\mathcal{V}_{i_{1} \bar{j}_{1}} \mathcal{V}_{i_{2} \bar{j}_{2}} \epsilon^{i_{1} i_{2}} \epsilon^{\bar{j}_{1} \bar{j}_{2}}=2\left(\left|\mathcal{V}_{1}\right|^{2}\left|\mathcal{V}_{2}\right|^{2}-\left|\mathcal{V}_{1} \cdot \overline{\mathcal{V}}_{\overline{2}}\right|^{2}\right)
$$

which can be recognized as (twice the) the squared norm of the $(\mathrm{SL}(2, \mathbb{C}) \sim \mathrm{SO}(3,1))$ vector $\mathcal{V}_{i \bar{j}}$. By respectively denoting with $\mathfrak{I}_{p}$ and $\widetilde{\mathfrak{G}}_{p}$ the dimension of a complete basis of $[\mathrm{SL}(p, \mathbb{C}) \times \mathrm{U}(r, s)]$-invariant polynomials and the orbit of the irrep. $(\mathbf{p}, \mathbf{r}+\mathbf{s})$ of $[\mathrm{SL}(p, \mathbb{C}) \times \mathrm{U}(r, s)]$ itself, the counting

$$
\mathfrak{I}_{p}=1
$$

is consistent with the general counting rule:

$$
\mathfrak{I}_{p}=2(r+s) p-\operatorname{dim}_{\mathbb{R}}\left(\widetilde{\mathfrak{G}}_{p}\right),
$$

because $\widetilde{\mathfrak{G}}_{p}$ generally is the direct product of the Riemannian symmetric non-compact coset $(\mathrm{SU}(p)=\operatorname{mcs}[\mathrm{SL}(p, \mathbb{C})])$

$$
\frac{\mathrm{SL}(p, \mathbb{C})}{\mathrm{SU}(p)}, \operatorname{dim}_{\mathbb{R}}=p^{2}-1
$$

and of a suitable non-compact form of the compact coset (3.7):

$$
\widetilde{\mathfrak{G}}_{p, \text { compact }}=\frac{\mathrm{SL}(p, \mathbb{C})}{\mathrm{SU}(p)} \times \frac{\mathrm{U}(r+s)}{\mathrm{U}(r+s-p)} \sim \frac{\mathrm{SL}(p, \mathbb{C}) \times \mathrm{U}(r+s)}{\mathrm{SU}(p) \times \mathrm{U}(r+s-p)}, \operatorname{dim}_{\mathbb{R}}=2(r+s) p-1 .
$$

It is immediate to realize that $\mathrm{SL}(p, \mathbb{C})$ cannot be the "horizontal" symmetry of a $p$-centered $\mathrm{BH}$ solution of the models under consideration. Indeed, the total symmetry $\mathrm{SL}(p, \mathbb{C}) \times \mathrm{U}(r+s)$ exhibits no invariants of order 2 in charges, as instead the symplectic product $\mathcal{W}(2.16)$ should generally be.

\section{$3.2 \mathrm{SL}_{h}(2, \mathbb{R}) \times \mathrm{U}(r, s)$}

The number and the structure of $p$-centered $(2 \leqslant p \leqslant r+s)$ algebraically independent duality-invariant homogeneous polynomials in the $\mathcal{N}=2$ minimally coupled theory have been already discussed in section 4.2.1 of [26]. We now give a unified treatment (holding for both the theories (3.1) and (3.2)) of both $p$-centered $(2 \leqslant p \leqslant r+s)$ "horizontal"- and duality- invariant polynomials.

As mentioned above, the 2-form field strengths and their dual (and thus the corresponding fluxes) sit in the complex fundamental irrep. $\mathbf{r}+\mathbf{s}$ of $G_{4}=\mathrm{U}(r, s)$. When 
considering a $p$-centered $\mathrm{BH}$ solution, the complex(ified) flux vector $\mathcal{Q}_{i}^{A}(A=1, \ldots, r+s$, $i=1, \ldots, p)$ sits in the bi-fundamental irrep. $(\mathbf{p}, \mathbf{r}+\mathbf{s})$ of the

$$
\text { "horizontal" } \times U \text {-duality group : } \mathrm{SL}_{h}(p, \mathbb{R}) \times \mathrm{U}(r, s) \text {. }
$$

As noticed in [26] for the $\mathcal{N}=2$ minimally coupled case, there are only $p^{2}$ algebraicallyindependent $\mathrm{U}(r, s)$-invariant homogeneous polynomials, all of order 2 in the fluxes, given by

$$
v_{i j} \equiv \mathcal{Q}_{i}^{A} \overline{\mathcal{Q}}_{j}^{\bar{B}} \eta_{A \bar{B}} \equiv \mathcal{Q}_{i} \cdot \overline{\mathcal{Q}}_{j}=\mathcal{S}_{i j}+\mathcal{W}_{i j}
$$

where "." denotes the scalar product determined by the pseudo-Euclidean metric $\eta_{A \bar{B}}$ of $\mathrm{U}(r, s)$, and

$$
\begin{aligned}
\mathcal{S}_{i j} & \equiv \mathcal{Q}_{(i} \cdot \overline{\mathcal{Q}}_{j)} \\
\mathcal{W}_{i j} & \equiv \mathcal{Q}_{[i} \cdot \overline{\mathcal{Q}}_{j]} .
\end{aligned}
$$

Note that, with respect to (3.4), the "horizontal" indices $i$ 's here belong to the real fundamental irrep. $\mathbf{p}$ of $\mathrm{SL}_{h}(p, \mathbb{R})$. By respectively denoting with $I_{p}$ and $\mathfrak{G}_{p}$ the dimension of a complete basis of duality invariant polynomials and the orbit of the irrep. $\mathbf{r}+\mathbf{s}$ of $G_{4}$, one obtains the very same counting given by eqs. (3.5)-(3.7).

Let us now consider the issue of "horizontal" invariants in the 2-centered $(p=2)$ case.

In this case, there are $p^{2}=4$ order- $2 \mathrm{U}(r, s)$-invariant polynomials $v_{i \bar{j}}(3.15)$, namely [26]:

$$
\begin{aligned}
\mathcal{S}_{11} & =\mathcal{Q}_{1} \cdot \overline{\mathcal{Q}}_{1} \equiv\left|\mathcal{Q}_{1}\right|^{2} \equiv I_{2}\left(\mathcal{Q}_{1}\right) \\
\mathcal{S}_{22} & =\left|\mathcal{Q}_{2}\right|^{2} \equiv I_{2}\left(\mathcal{Q}_{2}\right) \\
\mathcal{S}_{12} & =\operatorname{Re}\left(\mathcal{Q}_{1} \cdot \overline{\mathcal{Q}}_{2}\right) \equiv \mathbf{I}_{s} \\
\mathcal{W}_{12} & =i \operatorname{Im}\left(\mathcal{Q}_{1} \cdot \overline{\mathcal{Q}}_{2}\right) \equiv i \mathbf{I}_{a}=-i \mathcal{W}
\end{aligned}
$$

where $i, j=1,2$, and $I_{2}(\mathcal{Q})$ is the unique algebraically-independent 1 -centered $\mathrm{U}(r, s)$ invariant polynomial (homogeneous of order-2 in the charges $\mathcal{Q}$ 's; see e.g. [31, 36], and refs. therein):

$$
I_{2}(\mathcal{Q}) \equiv \mathcal{Q}^{A} \overline{\mathcal{Q}}^{\bar{B}} \eta_{A \bar{B}} \equiv \mathcal{Q} \cdot \overline{\mathcal{Q}} \equiv|\mathcal{Q}|^{2}
$$

Out of (3.18), one can easily determine that the "minimal degree" basis of homogeneous $\left[\mathrm{SL}_{h}(2, \mathbb{R}) \times \mathrm{U}(r+s)\right]$-invariant polynomials is composed by one invariant of order 2 , namely $\mathcal{W}(2.16)$, and by the following invariant of order $4\left(\epsilon^{12} \equiv 1\right)$ :

$$
\mathcal{I}_{1} \equiv \mathcal{S}_{i j} \mathcal{S}_{k l} \epsilon^{i k} \epsilon^{j l}=2\left(\mathcal{S}_{11} \mathcal{S}_{22}-\mathcal{S}_{12}^{2}\right)=2\left[I_{2}\left(\mathcal{Q}_{1}\right) I_{2}\left(\mathcal{Q}_{2}\right)-\mathbf{I}_{s}^{2}\right]
$$

In order to show this, we start and compute

$$
v_{i j} v_{k l} \epsilon^{i k} \epsilon^{j l}=\left(\mathcal{Q}_{i} \cdot \overline{\mathcal{Q}}_{j}\right)\left(\mathcal{Q}_{k} \cdot \overline{\mathcal{Q}}_{l}\right) \epsilon^{i k} \epsilon^{j l}=\left(\mathcal{S}_{i j}+\mathcal{W}_{i j}\right)\left(\mathcal{S}_{k l}+\mathcal{W}_{k l}\right) \epsilon^{i k} \epsilon^{j l}=\mathcal{V}_{1}+2 \mathcal{V}_{2}+\mathcal{V}_{3}
$$

By using the Schouten identities for $\mathrm{SL}_{h}(2, \mathbb{R})$

$$
\delta_{[a}^{e} \epsilon_{c d]}=0,
$$


it is immediate to obtain

$$
\begin{aligned}
& \mathcal{V}_{2} \equiv \mathcal{S}_{i j} \mathcal{W}_{k l} \epsilon^{i k} \epsilon^{j l}=0 \\
& \mathcal{V}_{3} \equiv \mathcal{W}_{i j} \mathcal{W}_{k l} \epsilon^{i k} \epsilon^{j l}=2 \mathcal{W}^{2}
\end{aligned}
$$

such that

$$
v_{i j} v_{k l} \epsilon^{i k} \epsilon^{j l}=2\left[I_{2}\left(\mathcal{Q}_{1}\right) I_{2}\left(\mathcal{Q}_{2}\right)-\mathbf{I}_{s}^{2}\right]+2 \mathcal{W}^{2}=2\left(\left|\mathcal{Q}_{1}\right|^{2}\left|\mathcal{Q}_{2}\right|^{2}-\left|\mathcal{Q}_{1} \cdot \overline{\mathcal{Q}}_{2}\right|^{2}\right) .
$$

By respectively denoting with $\widehat{\mathfrak{I}}_{2}$ and $\widehat{\mathfrak{G}}_{2}$ the dimension of a complete basis of $[\mathrm{SL}(2, \mathbb{R}) \times \mathrm{U}(r, s)]$-invariant polynomials and the orbit of the irrep. $(\mathbf{2}, \mathbf{r}+\mathbf{s})$ of $[\mathrm{SL}(2, \mathbb{R}) \times \mathrm{U}(r, s)]$ itself, the counting

$$
\widehat{\mathfrak{I}}_{2}=2
$$

is consistent with the general counting rule:

$$
\widehat{\mathfrak{I}}_{2}=4(r+s)-\operatorname{dim}_{\mathbb{R}}\left(\widehat{\mathfrak{G}}_{2}\right),
$$

because $\widehat{\mathfrak{G}}_{2}$ generally is the direct product of the Riemannian symmetric "horizontal" noncompact coset $(\mathrm{SO}(2)=\operatorname{mcs}[\mathrm{SL}(2, \mathbb{R})])$

$$
\frac{\mathrm{SL}(2, \mathbb{R})}{\mathrm{SO}(2)}, \operatorname{dim}_{\mathbb{R}}=2,
$$

and of a suitable non-compact form of the compact coset (3.7):

$$
\widehat{\mathfrak{G}}_{2, \text { compact }}=\frac{\mathrm{SL}(2, \mathbb{R})}{\mathrm{SO}(2)} \times \frac{\mathrm{U}(r+s)}{\mathrm{U}(r+s-2)} \sim \frac{\mathrm{SL}(2, \mathbb{R}) \times \mathrm{U}(r+s)}{\mathrm{SO}(2) \times \mathrm{U}(r+s-2)}, \operatorname{dim}_{\mathbb{R}}=4(r+s)-2 .
$$

Thus, at least in the $p=2$ case, an important feature of the models under consideration is that the "horizontal" sector coset (3.12) has a non-trivial stabilizer SO (2), differently e.g. from the CV (alias reducible symmetric) $[9,11]$ and from the irreducible symmetric $[10,11,21]$ models (also recall Footnote 4 ).

We leave the detailed investigation of the cases $p \geqslant 3$ for future further study.

\section{Remarks}

\subsection{On CV models}

We have given a complete analysis of the $\left[\mathrm{SL}_{v}(2, \mathbb{R}) \times \mathrm{SO}(m, n)\right]$ - (i.e. duality-) and $\left[\mathrm{SL}_{h}(2, \mathbb{R}) \times \mathrm{SL}_{v}(2, \mathbb{R}) \times \mathrm{SO}(m, n)\right]$ - (i.e. "horizontal") invariant homogeneous polynomials in Calabi-Vesentini (CV) $D=4$ supergravity models (cfr. eq. (1.1)), up to order 6 in the fluxes $\mathcal{Q}$ 's included. This analysis refines and completes the treatments of [9-11].

Consistent with analysis of [9] (and with the general results of [46]), a complete basis of homogeneous "horizontal" invariant polynomials for the CV models is given by (cfr. eq. (8.2) of [9], as well as the treatment of section 4 of [11])

$$
\left\{\mathcal{W}, \mathcal{X}, \mathbf{I}_{6}, \operatorname{Tr}\left(\mathfrak{I}_{0}^{2}\right)\right\},
$$


where the order-8 "horizontal" invariant polynomial $\operatorname{Tr}\left(\Im_{0}^{2}\right)$ is defined by eq. (4.9) of [9]. It is worth remarking that, as yielded by the general analysis of [46], besides being "of minimal order" in the fluxes $\mathcal{Q}_{1 \alpha}^{\Lambda}$ and $\mathcal{Q}_{2 \beta}^{\Sigma}$ of the two BH centers, the basis (4.1) is also freely generating the ring of "horizontal" invariant (homogeneous) polynomials: in other words, all other "horizontal" invariant polynomials are themselves polynomials in $\mathcal{W}, \mathcal{X}$, $\mathbf{I}_{6}$ and $\operatorname{Tr}\left(\mathfrak{I}_{0}^{2}\right)$, with no syzygial constraints. ${ }^{8}$

As for purely duality-invariant polynomials, we observe that their analysis at order- 6 in the fluxes has not been performed in section 2.3. This is due to the fact that, from the treatment given in section 5 of [9], it is known that a(n in general non-freely generating) complete basis "of minimal order" in the $\mathcal{Q}$ 's for the purely duality-invariant (homogeneous) polynomials is ${ }^{9}$ (cfr. eq. (1.15) of [11])

$$
\left\{\mathcal{W}, \mathcal{X}, I_{(a b c d)}\right\}
$$

and thus one does not need to seek for order-6 (and/or higher) purely duality-invariant homogeneous polynomials.

We would like also to point out that the non-generic example of CV model provided by the so-called $s t^{2}$ model is treated in section 6 of [9] and in section 4.2 and appendix $\mathrm{B}$ of [11]. Moreover, we recall that the so-called $t^{3}$ model (treated in section 7 of [9] and in section 5.2 and appendix B of [11]) is, as it is well known, an isolated case in the classification of symmetric special Kähler geometries (see e.g. [47], and [48] for a list of refs.); as such, it does not belong to the CV models (1.1). However, as shown in appendix B of [9], it can be reformulated in terms of a "constrained" CV symplectic frame.

\subsection{On pseudo-unitary $U$-duality}

On the other hand, the analysis carried out in section 3 (in turn refining the treatment given in section 4.2 .1 of [26]) yields that the (symmetric) $D=4$ supergravity models with $U$-duality group $G_{4}$ given by the pseudo-unitary group $\mathrm{U}(r, s)$ have a much simpler

\footnotetext{
${ }^{8}$ It should be pointed out that, due to the order-12 syzygial constraint given by eq. (5.6) of [9] (holding in all CV models), the "horizontal"-invariant basis (cfr. eq. (8.1) of [9], as well as the treatment of section 4 of $[11])$

is not freely generating.

For irreducible symmetric models [10, 21], due to the vanishing of $\mathcal{X}$ mentioned above, the complete basis "of minimal order" in the $\mathcal{Q}$ 's for "horizontal"-invariant (homogeneous) polynomials is given by

$$
\left\{\mathcal{W}, \mathbf{I}_{6}, \operatorname{Tr}\left(\mathfrak{I}_{0}^{2}\right), \operatorname{Tr}\left(\mathfrak{I}_{0}^{3}\right)\right\},
$$

and it is freely generating [46]. Therefore, apart from the peculiar case of the so-called $t^{3}$ model (treated in section 7 and appendix B of [9], as well as in section 5.2 of [11]), the $\mathcal{X}=0$ limit of the order-12 constraint given by eq. (5.6) of [9] does not hold in irreducible symmetric models.

${ }^{9}$ Of course, other choices are possible; see e.g. section 4 of [11].

For irreducible symmetric models, due to the vanishing of $\mathcal{X}$ mentioned above, the (in general non-freely generating) complete basis "of minimal order" in the $\mathcal{Q}$ 's for the purely duality-invariant (homogeneous) polynomials is given by eq. (1.16) of [11] (also in this case, other choice are of course possible; see e.g. section 5 of [11]).
} 
case study concerning the duality- and "horizontal" - invariant homogeneous polynomials constructed out of the BH fluxes' irrep., at least for $p=2$.

It is here worth commenting also about the $\mathcal{N}=2, D=4$ "magic" Maxwell-Einstein supergravity based on the irreducible cubic Jordan algebra $J_{3}^{\mathbb{C}}[49,50]$ (see also e.g. [13, 14] for a recent account) and on the $\mathcal{N}=5, D=4$ "pure" theory [51]. Despite the fact that their $U$-duality groups are suitable non-compact forms of the (special) pseudo-unitary group SU (6) (namely, $\mathrm{SU}(3,3)$ respectively $\mathrm{SU}(1,5)$ ), these theories do not belong to the class of models treated in section 3 . Indeed, they do not have an "extra" global symmetry $\mathrm{U}(1)$ under which their 2-form field strengths' fluxes are charged; this is also related to the fact that their magnetic and electric fluxes sit in a self-real irrep., namely the rank-3 completely antisymmetric $\mathbf{2 0}$ of SU (6) (and not in the complex fundamental irrep. 6 of the analogue would-be model of the type treated in section 3 ).

It should also be noticed that (non-compact forms of) $\mathbb{C} \mathbb{P}^{n}$ spaces as moduli spaces of string compactifications have appeared in the literature, either as particular subspaces of complex structure deformations of certain Calabi-Yau manifold $[52,53]$ or as moduli spaces of some asymmetric orbifolds of Type II superstrings [54]-[57], or of orientifolds [58].

Finally, we observe that the $D=4$ supergravity models considered in section 3 are not included in the analysis of [46]. In fact, only the "real (pseudo-orthogonal) analogues" of such models (in which the analogue of $\mathcal{W}_{i j}$ vanishes; see the treatment given in the second part of section 4 of [10]) can be found in table II of [46].

\subsection{On special geometry and "Generalized" groups of type $E_{7}$}

The sequence (3.1) and

$$
\frac{\mathrm{SL}(2, \mathbb{R})}{\mathrm{U}(1)} \times \frac{\mathrm{SO}(2, n-2)}{\mathrm{SO}(2) \times \mathrm{SO}(n-2)}, n \geqslant 3,
$$

related to the case $m=2$ of (1.1), are the unique sequences of symmetric non-compact spaces in the special Kähler geometry (SKG) of $\mathcal{N}=2, D=4$ vector multiplets (see e.g. [12, 37-39], and refs. therein).

Here we would like to discuss the characterization of SKG in terms of a suitable "generalization" of the groups of type $E_{7}$ [29] (for some preliminary discussion, see section 4 of [28]).

As obtained in [24] (see eq. (5.36) therein), the following real function, which we dub "entropy functional", can be defined on the vector multiplets' scalar manifold ${ }^{10} \mathrm{M}$ :

$$
\mathbb{I}_{4}=\left(|Z|^{2}-Z_{i} \bar{Z}^{i}\right)^{2}+\frac{2}{3} i\left(Z \bar{C}_{\overline{i j k}} Z^{\bar{i}} Z^{\bar{j}} Z^{\bar{k}}-\bar{Z} C_{i j k} \bar{Z}^{i} \bar{Z}^{j} \bar{Z}^{k}\right)-g^{i \bar{i}} C_{i j k} \bar{C}_{\bar{i} \bar{l} \bar{m}} \bar{Z}^{j} \bar{Z}^{k} Z^{\bar{l}} Z^{\bar{m}}
$$

$Z$ is the central extension of $\mathcal{N}=2, D=4$ local supersymmetry algebra, and $Z_{i} \equiv D_{i} Z$ are the so-called "matter charges" $\left(D_{i}\right.$ stands for the Kähler-covariant differential operator; see e.g. [40] and [12] for notation and further elucidation):

$$
Z \equiv \mathcal{Q}^{M} V^{N} \mathbb{C}_{M N} ; Z_{i} \equiv \mathcal{Q}^{M} V_{i}^{N} \mathbb{C}_{M N},
$$

\footnotetext{
${ }^{10}$ Note that the expression (4.4) is independent on the choice of the symplectic frame and manifestly invariant under diffeomorphisms in $\mathbf{M}$.
} 
with $V^{M}$ denoting the vector of covariantly-holomorphic symplectic sections of SKG, and $V_{i}^{M} \equiv D_{i} V^{M}$. Furthermore, $C_{i j k}$ is the rank-3, completely symmetric, covariantly holomorphic tensor of SKG (with Kähler weights $(2,-2)$ ) (see e.g. [41, 42]):

$$
\begin{aligned}
C_{i j k} & \equiv \mathbb{C}_{M N}\left(D_{i} D_{j} V^{M}\right) D_{k} V^{N}=-i g_{i \bar{l}} \bar{f}_{\Lambda}^{\bar{l}} D_{j} D_{k} L^{\Lambda}=D_{i} D_{j} D_{k} \mathcal{S}=e^{K} W_{i j k} ; \\
\bar{f}_{\Lambda}^{\bar{l}}\left(\bar{D}_{\bar{s}}^{\Lambda}\right) & \equiv \delta_{\bar{s}}^{\bar{l}}, \mathcal{S} \equiv-i L^{\Lambda} L^{\Sigma^{\Sigma}} \operatorname{Im}\left(F_{\Lambda \Sigma}\right), \bar{\partial}_{\bar{l}} W_{i j k}=0 ; \\
\bar{D}_{\bar{i}} C_{j k l} & =0 ; \\
D_{[i} C_{j] k l} & =0,
\end{aligned}
$$

the last property being a consequence, through the covariant holomorphicity of $C_{i j k}$ and the SKG constraint on the Riemann tensor (see e.g. [41-43])

$$
R_{j \bar{k} l \bar{m}}=-g_{j \bar{k}} g_{l \bar{m}}-g_{j \bar{m}} g_{l \bar{k}}+g^{i \bar{i}} C_{i j l} \bar{C}_{\overline{i k} \bar{m}}
$$

of the Bianchi identities satisfied by the Riemann tensor $R_{i \bar{j} k \bar{l}}$.

Furthermore, $\mathbb{I}_{4}$ is an order-4 homogeneous polynomial in the fluxes $\mathcal{Q}$; this allows for the definition of the $\mathcal{Q}$-independent rank-4 completely symmetric tensor $\Omega_{M N P Q}$ [28], whose general expression we explicitly compute here:

$$
\begin{aligned}
& \Omega_{M N P Q} \equiv 2 \frac{\partial^{4} \mathbb{I}_{4}}{\partial \mathcal{Q}^{(M} \partial \mathcal{Q}^{N} \partial \mathcal{Q}^{P} \partial \mathcal{Q}^{Q)}} \\
& =2 V_{(M} V_{N} \bar{V}_{P} \bar{V}_{Q)}+2 V_{i \mid(M} \bar{V}_{N}^{i} V_{j \mid P} \bar{V}_{Q)}^{j}-4 V_{(M} \bar{V}_{N} V_{i \mid P} \bar{V}_{Q)}^{i} \\
& +\frac{4}{3} i\left(\bar{C}_{\overline{i j k}} V_{(M} V_{N}^{\bar{i}} V_{P}^{\bar{j}} V_{Q}^{\bar{k}}-C_{i j k} \bar{V}_{(M} \bar{V}_{N}^{i} \bar{V}_{P}^{j} \bar{V}_{Q}^{k}\right) \\
& -2 g^{i \bar{i}} C_{i j k} \bar{C}_{\bar{i} \bar{l} \bar{m}} \bar{V}_{(M}^{j} \bar{V}_{N}^{k} V_{P}^{\bar{l}} V_{Q)}^{\bar{m}} \text {. } \\
& =2 V_{(M} V_{N} \bar{V}_{P} \bar{V}_{Q)}+2 V_{i \mid(M} \bar{V}_{N}^{i} V_{j \mid P} \bar{V}_{Q)}^{j}-4 V_{(M} \bar{V}_{N} V_{i \mid P} \bar{V}_{Q)}^{i} \\
& +\frac{2}{3}\left(V_{(M} V_{N}^{\bar{i}} V_{P}^{\bar{j}} \bar{D}_{\bar{i}} \bar{V}_{\bar{j} \mid Q}+\bar{V}_{(M} \bar{V}_{N}^{i} \bar{V}_{P}^{j} D_{i} V_{j \mid Q}\right) \\
& -2 g^{i \bar{i}} \bar{V}_{(M}^{j} V_{N}^{\bar{l}} D_{i} V_{j \mid N} \bar{D}_{\bar{i}} \bar{V}_{\bar{l} \mid Q)},
\end{aligned}
$$

where the SKG defining relation (see e.g. [41-43])

$$
D_{i} D_{j} V^{M} \equiv D_{i} V_{j}^{M}=i C_{i j k} \bar{V}^{k \mid M}
$$

has been used in order to recast (4.8) in terms of $V^{M}, V_{i}^{M}$ and $D_{i} V_{j}^{M}$ only.

Some further elaborations are possible; e.g., by using (4.7), $\mathbb{I}_{4}$ (4.4) and $\Omega_{M N P Q}$ (4.9) can respectively be rewritten as

$$
\begin{aligned}
\mathbb{I}_{4}= & |Z|^{4}-\left(Z_{i} \bar{Z}^{i}\right)^{2}-2|Z|^{2} Z_{i} \bar{Z}^{i}+\frac{2}{3} i\left(Z \bar{C} \overline{i j k} Z^{\bar{i}} Z^{\bar{j}} Z^{\bar{k}}-\bar{Z} C_{i j k} \bar{Z}^{i} \bar{Z}^{j} \bar{Z}^{k}\right)-\mathcal{R} \\
\Omega_{M N P Q}= & 2 V_{(M} V_{N} \bar{V}_{P} \bar{V}_{Q)}-2 V_{i \mid(M} \bar{V}_{N}^{i} V_{j \mid P} \bar{V}_{Q)}^{j}-4 V_{(M} \bar{V}_{N} V_{i \mid P} \bar{V}_{Q)}^{i} \\
& +\frac{2}{3}\left(V_{(M} V_{N}^{\bar{i}} V_{P}^{\bar{j}} \bar{D}_{\bar{i}} \bar{V}_{\bar{j} \mid Q}+\bar{V}_{(M} \bar{V}_{N}^{i} \bar{V}_{P}^{j} D_{i} V_{j \mid Q}\right)-R_{M N P Q}
\end{aligned}
$$


where the sectional curvature of matter charges (cfr. eq. (5.3) of [44]; also note that (4.13) is different from the definition given by eq. (3.1.1.2.11) of [45]))

$$
\mathcal{R} \equiv R_{i \bar{j} k \bar{l}} \bar{Z}^{i} Z^{\bar{j}} \bar{Z}^{k} Z^{\bar{l}},
$$

and the corresponding rank-4 completely symmetric tensor

$$
R_{M N P Q} \equiv \frac{\partial^{4} \mathcal{R}}{\partial \mathcal{Q}^{(M} \partial \mathcal{Q}^{N} \partial \mathcal{Q}^{P} \partial \mathcal{Q}^{Q)}}=R_{i \bar{j} k \bar{l}} \bar{V}_{(M}^{i} V_{N}^{\bar{j}} \bar{V}_{P}^{k} V_{Q)}^{\bar{l}},
$$

have been introduced. Note that $R_{M N P Q}$ can be regarded as the completely symmetric part of the "symplectic pull-back" (through the symplectic sections $V_{i}^{M}$ ) of the Riemann tensor $R_{i \bar{j} k \bar{l}}$ of $\mathbf{M}$.

Thus, SKG can be associated to a generalization of the class of groups of type $E_{7}$ [29], based on $\mathbb{I}_{4}$ and the corresponding (generally field-dependent, non-constant) $\Omega$-structure:

$$
\text { SKG }:\left\{\begin{array}{l}
\Omega_{M N P Q}: D_{i} \Omega_{M N P Q}=\partial_{i} \Omega_{M N P Q} \neq 0 \\
\mathbb{I}_{4} \equiv \frac{1}{2} \Omega_{M N P Q} \mathcal{Q}^{M} \mathcal{Q}^{N} \mathcal{Q}^{P} \mathcal{Q}^{Q} \Rightarrow D_{i} \mathbb{I}_{4}=\partial_{i} \mathbb{I}_{4} \neq 0 .
\end{array}\right.
$$

Symmetric Kähler spaces have a covariantly constant Riemann tensor:

$$
D_{i} R_{j \bar{k} l \bar{m}}=0 .
$$

Within SKG, through the constraint (4.7), this implies the covariant constancy of the $C$-tensor (4.6):

$$
D_{(i} C_{j) k l}=D_{(i} C_{j k l)}=0,
$$

which in turn yields the relation:

$$
C_{p(k l} C_{i j) n} g^{n \bar{n}} g^{p \bar{p}} \bar{C}_{\overline{n p m}}=\frac{4}{3} g_{(l \mid \bar{m}} C_{\mid i j k)} \Leftrightarrow g^{n \bar{n}} R_{(i|\bar{m}| j \mid \bar{n}} C_{n \mid k l)}=-\frac{2}{3} g_{(i \mid \bar{m}} C_{\mid j k l)} .
$$

Equivalently, symmetric SK manifolds can be characterized by stating that their $\Omega_{M N P Q}$ is is independent on the scalar fields themselves, and it matches the $\mathbb{K}$-tensor $\mathbb{K}_{M N P Q}$ defining the rank-4 invariant $\mathbb{K}$-structure of the corresponding $U$-duality group of type $E_{7}$ [29] (see also e.g. [21], and refs. therein). Consequently, the corresponding "entropy functional" $\mathbb{I}_{4}(4.4)$ is independent on the scalar fields themselves, and it is thus a constant function in M, given by the 1-centered limit of the Dixmier tensor $I_{a b c d}(2.43)$, which is nothing but the unique algebraically-independent 1 -centered $U$-duality invariant polynomial $I_{4}$ :

$$
\begin{gathered}
\text { symmetric SKG } \\
\left(U \text {-duality group } G_{4} \text { is of type } E_{7}\right)
\end{gathered} \Rightarrow\left\{\begin{array}{l}
\Omega_{M N P Q}=\mathbb{K}_{M N P Q} \Rightarrow D_{i} \Omega_{M N P Q}=\partial_{i} \Omega_{M N P Q}=0 ; \\
\mathbb{I}_{4}=I_{4} \equiv \frac{1}{2} \mathbb{K}_{M N P Q} \mathcal{Q}^{M} \mathcal{Q}^{N} \mathcal{Q}^{P} \mathcal{Q}^{Q} \Rightarrow D_{i} \mathbb{I}_{4}=\partial_{i} \mathbb{I}_{4}=0 .
\end{array}\right.
$$

In turn, within symmetric $\mathrm{SKG}$, the pseudo-unitary $U$-duality group $\mathrm{U}(1, s)$ (corresponding to $\mathcal{N}=2$ minimally coupled Maxwell-Einstein theory $[30,31])$ is "degenerate", in the sense that the corresponding $I_{4}$ actually is the square of the order-2 $\mathrm{U}(1, s)$-invariant polynomial 
$I_{2}$ (3.19). Indeed, $\mathcal{N}=2$ minimally coupled supergravity is characterized by ${ }^{11} C_{i j k}=0$, which plugged into (4.4) (by taking (4.19) into account) yields:

$$
\underset{G_{4}=\mathrm{U}(1, s)}{\text { symmetric } \mathrm{SKG}} \Rightarrow\left\{\begin{array}{l}
\Omega_{M N P Q}=\mathbb{K}_{M N P Q} \Rightarrow D_{i} \Omega_{M N P Q}=\partial_{i} \Omega_{M N P Q}=0 ; \\
C_{i j k}=0 ; \\
\mathbb{I}_{4}=I_{4}=\left(|Z|^{2}-Z_{i} \bar{Z}^{i}\right)^{2}=\frac{1}{4} I_{2}^{2} \Rightarrow D_{i} \mathbb{I}_{4}=\partial_{i} \mathbb{I}_{4}=0,
\end{array}\right.
$$

where the normalization of [26] (see eq. (2.15) therein) has been adopted.

We conclude by recalling that, as noticed in [24] and in [28], the "entropic functional" $\mathbb{I}_{4}$ (4.4) is related to the geodesic potential defined in the $D=4 \rightarrow 3$ dimensional reduction of the considered $\mathcal{N}=2$ theory. Under such a reduction, the $D=4$ vector multiplets' SK manifold $\mathbf{M}\left(\operatorname{dim}_{\mathbb{C}}=n_{V}\right)$ enlarges to a special quaternionic Kähler manifold $\mathfrak{M}$ $\left(\operatorname{dim}_{\mathbb{H}}=n_{V}+1\right)$ given by $c$-map $[47,59]$ of $\mathbf{M}$ itself : $\mathfrak{M}=c(\mathbf{M})$. By specifying eq. (4.4) in the " $4 D / 5 D$ special coordinates" " symplectic frame, $\mathbb{I}_{4}$ matches the opposite of the function $h$ defined by eq. (4.42) of [60], within the analysis of special quaternionic Kähler geometry. This relation can be strengthened by observing that the tensor $\Omega_{M N P Q}$ given by (4.8)-(4.9) is proportional to the $\Omega$-tensor of quaternionic geometry, related to the quaternionic Riemann tensor by eq. (15) of [61]; for further comments, see [28].

\section{Acknowledgments}

A. M. gratefully acknowledges enlightening discussions with Laurent Manivel and Bert Van Geemen, who also drew to his attention [46] and [27], respectively.

A. Y. would like to thank CERN Theory Division for kind hospitality.

The work of S. F. is supported by the ERC Advanced Grant no. 226455, "Supersymmetry, Quantum Gravity and Gauge Fields" (SUPERFIELDS), and in part by DOE Grant DE-FG03-91ER40662.

The work of A. Y. is supported by the ERC Advanced Grant no. 226455, "Supersymmetry, Quantum Gravity and Gauge Fields" (SUPERFIELDS).

Open Access. This article is distributed under the terms of the Creative Commons Attribution License which permits any use, distribution and reproduction in any medium, provided the original author(s) and source are credited.

\section{References}

[1] A. Sen, Walls of marginal stability and dyon spectrum in $\mathcal{N}=4$ supersymmetric string theories, JHEP 05 (2007) 039 [hep-th/0702141] [INSPIRE].

\footnotetext{
${ }^{11}$ By plugging $C_{i j k}=0$ into (4.10) and recalling definition (4.5), one obtains the Kähler-covariant antiholomorphicity of $V_{j}^{M}$, and thus of $Z_{j}$ :

$$
D_{i} V_{j}^{M}=0 \Rightarrow D_{i} Z_{j}=0 .
$$
}


[2] A. Sen, Two centered black holes and $\mathcal{N}=4$ dyon spectrum, JHEP 09 (2007) 045 [arXiv:0705.3874] [INSPIRE].

[3] A. Sen, $\mathcal{N}=8$ dyon partition function and walls of marginal stability, JHEP 07 (2008) 118 [arXiv:0803.1014] [INSPIRE].

[4] A. Sen, Wall crossing formula for $\mathcal{N}=4$ dyons: a macroscopic derivation, JHEP 07 (2008) 078 [arXiv: 0803.3857] [INSPIRE].

[5] J.R. David, On walls of marginal stability in $\mathcal{N}=2$ string theories, JHEP 08 (2009) 054 [arXiv: 0905.4115] [INSPIRE].

[6] E. Cremmer and B. Julia, The $\mathcal{N}=8$ supergravity theory. 1. The Lagrangian, Phys. Lett. B 80 (1978) 48 [INSPIRE].

[7] E. Cremmer and B. Julia, The SO(8) supergravity, Nucl. Phys. B 159 (1979) 141 [INSPIRE].

[8] C. Hull and P. Townsend, Unity of superstring dualities, Nucl. Phys. B 438 (1995) 109 [hep-th/9410167] [INSPIRE].

[9] S. Ferrara, A. Marrani, E. Orazi, R. Stora and A. Yeranyan, Two-center black holes duality-invariants for STU model and its lower-rank descendants,

J. Math. Phys. 52 (2011) 062302 [arXiv:1011.5864] [INSPIRE].

[10] L. Andrianopoli, R. D'Auria, S. Ferrara, A. Marrani and M. Trigiante, Two-centered magical charge orbits, JHEP 04 (2011) 041 [arXiv:1101.3496] [INSPIRE].

[11] A. Ceresole, S. Ferrara, A. Marrani and A. Yeranyan, Small black hole constituents and horizontal symmetry, JHEP 06 (2011) 078 [arXiv:1104.4652] [INSPIRE].

[12] L. Andrianopoli et al., $\mathcal{N}=2$ supergravity and $\mathcal{N}=2$ super Yang-Mills theory on general scalar manifolds: symplectic covariance, gaugings and the momentum map,

J. Geom. Phys. 23 (1997) 111 [hep-th/9605032] [InSPIRE].

[13] L. Borsten, M. Duff, S. Ferrara, A. Marrani and W. Rubens, Small orbits, arXiv:1108.0424 [INSPIRE].

[14] L. Borsten, M. Duff, S. Ferrara, A. Marrani and W. Rubens, Explicit orbit classification of reducible Jordan algebras and Freudenthal triple systems, arXiv:1108.0908 [INSPIRE].

[15] E. Calabi and E. Vesentini, On compact, locally symmetric Kähler manifolds, Ann. Math. 71 (1960) 472.

[16] A. Ceresole, R. D'Auria, S. Ferrara and A. Van Proeyen, Duality transformations in supersymmetric Yang-Mills theories coupled to supergravity, Nucl. Phys. B 444 (1995) 92 [hep-th/9502072] [INSPIRE].

[17] M. Cvetič and D. Youm, Dyonic BPS saturated black holes of heterotic string on a six torus, Phys. Rev. D 53 (1996) 584 [hep-th/9507090] [INSPIRE].

[18] M. Duff, J.T. Liu and J. Rahmfeld, Four-dimensional string-string-string triality, Nucl. Phys. B 459 (1996) 125 [hep-th/9508094] [INSPIRE].

[19] M. Cvetič and A.A. Tseytlin, General class of BPS saturated dyonic black holes as exact superstring solutions, Phys. Lett. B 366 (1996) 95 [hep-th/9510097] [INSPIRE].

[20] M. Cvetič and A.A. Tseytlin, Solitonic strings and BPS saturated dyonic black holes, Phys. Rev. D 53 (1996) 5619 [Erratum ibid. D 55 (1997) 3907] [hep-th/9512031] [INSPIRE]. 
[21] A. Marrani, E. Orazi and F. Riccioni, Exceptional reductions, J. Phys. A 44 (2011) 155207 [arXiv: 1012.5797] [INSPIRE].

[22] S. Ferrara and J.M. Maldacena, Branes, central charges and U duality invariant BPS conditions, Class. Quant. Grav. 15 (1998) 749 [hep-th/9706097] [INSPIRE].

[23] L. Andrianopoli, R. D'Auria and S. Ferrara, U invariants, black hole entropy and fixed scalars, Phys. Lett. B 403 (1997) 12 [hep-th/9703156] [INSPIRE].

[24] B.L. Cerchiai, S. Ferrara, A. Marrani and B. Zumino, Duality, entropy and ADM mass in supergravity, Phys. Rev. D 79 (2009) 125010 [arXiv:0902.3973] [InSPIRE].

[25] L. Andrianopoli, R. D'Auria, S. Ferrara and M. Trigiante, Fake superpotential for large and small extremal black holes, JHEP 08 (2010) 126 [arXiv: 1002.4340] [INSPIRE].

[26] S. Ferrara, A. Marrani and E. Orazi, Split attractor flow in $\mathcal{N}=2$ minimally coupled supergravity, Nucl. Phys. B 846 (2011) 512 [arXiv:1010.2280] [INSPIRE].

[27] J. Dixmier, On the projective invariants of quartic plane curves, Adv. Math. 64 (1987) 279.

[28] S. Ferrara, A. Marrani and A. Yeranyan, Freudenthal duality and generalized special geometry, Phys. Lett. B 701 (2011) 640 [arXiv:1102.4857] [INSPIRE].

[29] R.B. Brown, Groups of type E $E_{7}, J$. Reine Angew. Math. 236 (1969) 79.

[30] J. Luciani, Coupling of $O(2)$ supergravity with several vector multiplets, Nucl. Phys. B 132 (1978) 325 [inSPIRE].

[31] S. Ferrara, A. Gnecchi and A. Marrani, $D=4$ attractors, effective horizon radius and fake supergravity, Phys. Rev. D 78 (2008) 065003 [arXiv:0806.3196] [InSPIRE].

[32] L. Castellani et al., The complete $\mathcal{N}=3$ matter coupled supergravity, Nucl. Phys. B 268 (1986) 317 [InSPIRE].

[33] M. Duff and S. Ferrara, Generalized mirror symmetry and trace anomalies, Class. Quant. Grav. 28 (2011) 065005 [arXiv: 1009.4439] [InSPIRE].

[34] S. Ferrara and A. Marrani, Matrix norms, BPS bounds and marginal stability in $\mathcal{N}=8$ supergravity, JHEP 12 (2010) 038 [arXiv:1009.3251] [INSPIRE].

[35] S. Ferrara, J. Scherk and B. Zumino, Algebraic properties of extended supergravity theories, Nucl. Phys. B 121 (1977) 393 [INSPIRE].

[36] L. Andrianopoli, R. D'Auria and S. Ferrara, U invariants, black hole entropy and fixed scalars, Phys. Lett. B 403 (1997) 12 [hep-th/9703156] [INSPIRE].

[37] E. Cremmer et al., Vector multiplets coupled to $\mathcal{N}=2$ supergravity: superHiggs effect, flat potentials and geometric structure, Nucl. Phys. B 250 (1985) 385 [INSPIRE].

[38] E. Cremmer and A. Van Proeyen, Classification of Kähler manifolds in $\mathcal{N}=2$ vector multiplet supergravity couplings, Class. Quant. Grav. 2 (1985) 445 [INSPIRE].

[39] A. Strominger, Special geometry, Commun. Math. Phys. 133 (1990) 163 [INSPIRE].

[40] A. Ceresole, R. D'Auria and S. Ferrara, The symplectic structure of $\mathcal{N}=2$ supergravity and its central extension, Nucl. Phys. Proc. Suppl. 46 (1996) 67 [hep-th/9509160] [InSPIRE].

[41] L. Castellani, R. D'Auria and S. Ferrara, Special geometry without special coordinates, Class. Quant. Grav. 7 (1990) 1767 [INSPIRE]. 
[42] L. Castellani, R. D'Auria and S. Ferrara, Special Kähler geometry: an intrinsic formulation from $\mathcal{N}=2$ space-time supersymmetry, Phys. Lett. B 241 (1990) 57 [INSPIRE].

[43] B. Craps, F. Roose, W. Troost and A. Van Proeyen, What is special Kähler geometry?, Nucl. Phys. B 503 (1997) 565 [hep-th/9703082] [INSPIRE].

[44] S. Bellucci, A. Marrani and R. Roychowdhury, On quantum special Kähler geometry, Int. J. Mod. Phys. A 25 (2010) 1891 [arXiv:0910.4249] [InSPIRE].

[45] S. Bellucci, S. Ferrara, R. Kallosh and A. Marrani, Extremal black hole and flux vacua attractors, Lect. Notes Phys. 755 (2008) 115 [arXiv:0711.4547] [INSPIRE].

[46] V.G. Kac, Some remarks on nilpotent orbits, J. Algebra 64 (1980) 190.

[47] S. Cecotti, S. Ferrara and L. Girardello, Geometry of type II superstrings and the moduli of superconformal field theories, Int. J. Mod. Phys. A 4 (1989) 2475 [InSPIRE].

[48] S. Ferrara and A. Marrani, Symmetric spaces in supergravity, in "Symmetry in Mathematics and Physics", D. Babbitt, V. Vyjayanthi and R. Fioresi eds., Contemporary Mathematics 490, American Mathematical Society, Providence U.S.A. (2009) [arXiv:0808.3567] [INSPIRE].

[49] M. Günaydin, G. Sierra and P. Townsend, Exceptional supergravity theories and the MAGIC square, Phys. Lett. B 133 (1983) 72 [INSPIRE].

[50] M. Günaydin, G. Sierra and P. Townsend, The geometry of $\mathcal{N}=2$ Maxwell-Einstein supergravity and Jordan algebras, Nucl. Phys. B 242 (1984) 244 [INSPIRE].

[51] B. de Wit and H. Nicolai, Extended supergravity with local $\mathrm{SO}(5)$ invariance, Nucl. Phys. B 188 (1981) 98 [inSPIRE].

[52] A. Ceresole, R. D'Auria and T. Regge, Duality group for Calabi-Yau 2 moduli space, Nucl. Phys. B 414 (1994) 517 [hep-th/9307151] [INSPIRE].

[53] L.J. Dixon, V. Kaplunovsky and J. Louis, On effective field theories describing $(2,2)$ vacua of the heterotic string, Nucl. Phys. B 329 (1990) 27 [INSPIRE].

[54] S. Ferrara and C. Kounnas, Extended supersymmetry in four-dimensional type II strings, Nucl. Phys. B 328 (1989) 406 [InSPIRE].

[55] S. Ferrara and P. Fré, Type II superstrings on twisted group manifolds and their heterotic counterparts, Int. J. Mod. Phys. A 5 (1990) 989 [InSPIRE].

[56] A. Dabholkar and J.A. Harvey, String islands, JHEP 02 (1999) 006 [hep-th/9809122] [INSPIRE].

[57] C. Kounnas and A. Kumar, BPS states in $\mathcal{N}=3$ superstrings, Nucl. Phys. B 511 (1998) 216 [hep-th/9709061] [inSPIRE].

[58] A.R. Frey and J. Polchinski, $\mathcal{N}=3$ warped compactifications, Phys. Rev. D 65 (2002) 126009 [hep-th/0201029] [INSPIRE].

[59] S. Ferrara and S. Sabharwal, Quaternionic manifolds for type II superstring vacua of Calabi-Yau spaces, Nucl. Phys. B 332 (1990) 317 [INSPIRE].

[60] B. de Wit, F. Vanderseypen and A. Van Proeyen, Symmetry structure of special geometries, Nucl. Phys. B 400 (1993) 463 [hep-th/9210068] [INSPIRE].

[61] J. Bagger and E. Witten, Matter couplings in $\mathcal{N}=2$ supergravity, Nucl. Phys. B 222 (1983) 1 [inSPIRE]. 